\title{
The IncRNA PVT1 regulates nasopharyngeal carcinoma cell proliferation via activating the KAT2A acetyltransferase and stabilizing HIF-1a
}

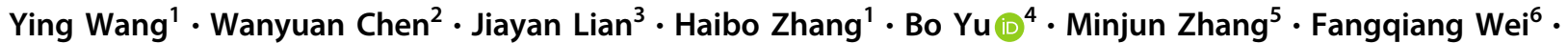

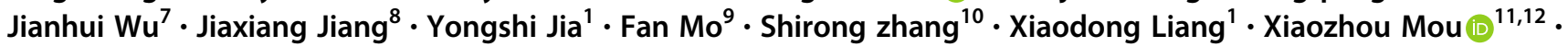 \\ Jianming Tang ${ }^{1}$
}

Received: 9 April 2019 / Revised: 13 June 2019 / Accepted: 19 June 2019 / Published online: 18 July 2019

(c) The Author(s) 2019. This article is published with open access

\begin{abstract}
Long noncoding RNAs (lncRNAs) play important roles in regulating the development and progression of many cancers. However, the clinical significance of specific lncRNAs in the context of nasopharyngeal carcinoma (NPC) and the molecular mechanisms by which they regulate this form of cancer remain largely unclear. In this study we found that the lncRNA PVT1 was upregulated in NPC, and that in patients this upregulation was associated with reduced survival. RNA sequencing revealed that PVT1 was responsible for regulating NPC cell proliferation and for controlling a hypoxia-related phenotype in these cells. PVT1 knockdown reduced NPC cell proliferation, colony formation, and tumorigenesis in a subcutaneous mouse xenograft model systems. We further found that PVT1 serves as a scaffold for the chromatin modification factor KAT2A, which mediates histone 3 lysine 9 acetylation (H3K9), recruiting the nuclear receptor binding protein TIF1 $\beta$ to activate NF90 transcription, thereby increasing HIF-1 $\alpha$ stability and promoting a malignant phenotype in NPC cells. Overexpression of NF90 or HIF-1 $\alpha$ restored the proliferation in cells that had ceased proliferating due to PVT1 or KAT2A depletion. Conversely, overexpression of active KAT2A or TIF1 $\beta$, but not of KAT2A acetyltransferase activity-deficient mutants or TIF1 $\beta$ isoforms lacking H3K9ac binding sites, promoted a PVT1-mediated increase in NF90 transcription, as well as increased HIF-1 $\alpha$ stability and cell proliferation. PVT1 knockdown enhanced the radiosensitization effect in NPC cells via inhibiting binding between $\mathrm{H} 3 \mathrm{~K} 9 \mathrm{ac}$ and TIF1 $\beta$ in a manner. Taken together, our results demonstrate that PVT1 serves an oncogenic role and plays an important role in radiosensitivity in malignant NPC via activating the KAT2A acetyltransferase and stabilizing HIF- $1 \alpha$.
\end{abstract}

These authors contributed equally: Ying Wang, Wanyuan Chen, Jiayan Lian, Haibo Zhang, Bo Yu

Edited by H. Ichijo

Supplementary information The online version of this article (https:// doi.org/10.1038/s41418-019-0381-y) contains supplementary material, which is available to authorized users.

Xiaodong Liang

lxdctopone@sina.com

$\triangle$ Xiaozhou Mou

mouxz@zju.edu.cn

$\triangle$ Jianming Tang 15900792812@163.com

Extended author information available on the last page of the article

\section{Introduction}

Nasopharyngeal carcinoma (NPC), which is a form of cancer arising from the epithelium of the nasopharynx, remains highly prevalent, particularly in Southeast Asia and Southern China [1, 2]. Although intensity-modulated radiation advances in NPC treatment, tumor proliferation, and growth for NPC patients remain to be the important cause of treatment failure and cancer-related death [3, 4]. Recently, an increasing body of evidence has suggested that long noncoding RNAs (lncRNAs) are involved in tumorigenesis through regulating histone modification [5, 6]. However, the mechanisms that account for it remain to be elucidated.

Plasmacytoma variant translocation 1 (PVT1) is a lncRNA that has been found to serve an oncogenic role in a variety of malignant tumors. PVT1 was first discovered to 
be frequently translocated in mouse models of plasmacytoma, ultimately contributing to carcinogenesis in these models [7, 8]. Recent evidence further indicates that PVT1 exhibits aberrant expression in nonsmall-cell lung cancer [9-11], cervical cancer [10], colorectal cancer [12], and gastric cancer [13, 14]. Moreover, PVT1 expression is significantly linked to patient survival in those with colorectal [15], lung [16], and breast cancer [17]. PVT1 has been shown to directly bind and stabilize the KLF5 proteins in breast cancer [17]. Enhancer of zeste homolog 2 (EZH2), a major histone methyltransferase, plays an essential role in tumor regulation via trimethylating lysine 27 on histone $\mathrm{H} 3$. EZH2 forms a molecular complex with PVT1 to function as an repressive driver of p15 and p16 in gastric cancer [18]. PVT1 is also transcriptionally activated by FOXM1 in gastric cancer [19]. Furthermore, PVT1 induces radioresistance by influencing cell apoptosis and DNA repair in NPC [20]. However, the specific biological importance and clinical significance of PVT1 in NPC progression remains to be established.

In the present study, we found that PVT1 was upregulated in NPC, and that it predicted poor survival in patients. PVT1 promoted this NPC cell proliferation via activating the KAT2A H3K9 acetyltransferase and TIF1 $\beta$ activity to activate NF90 transcription and increase HIF- $1 \alpha$ stability. Interestingly, PVT1 contributed to the radiosensitization effect in NPC cells by enhancing the binding of H3K9ac and TIF1 $\beta$ in a manner. Collectively, our results establish a new regulatory mechanism by which PVT1 promotes NPC progression, providing a potential therapeutic target and prognostic factor for NPC.

\section{Results}

\section{The PVT1 IncRNA is upregulated in NPC and is associated with a poor prognosis in patients}

To identify the functions of PVT1 in NPC progression, we first analyzed PVT1 expression in the NP69 immortalized nasopharyngeal epithelial cell line and in five NPC cell lines (HNE-1, C666-1, CNE-1, SUNE-1, and CNE-2). Interestingly, we found that the PVT1 expression level was higher in NPC cell lines than that in NP69 cells (Fig. 1a). We next examined PVT1 expression in ten freshly frozen normal nasopharyngeal specimens and in ten clinical NPC tumor samples. As shown in Fig. 1b, compared with the normal nasopharyngeal epithelial specimens, PVT1 expression was markedly elevated in NPC tumors. To further confirm this finding, we obtained gene expression data from the microarray datasets GSE12452 [21] and GSE64634 [22], and examined PVT1
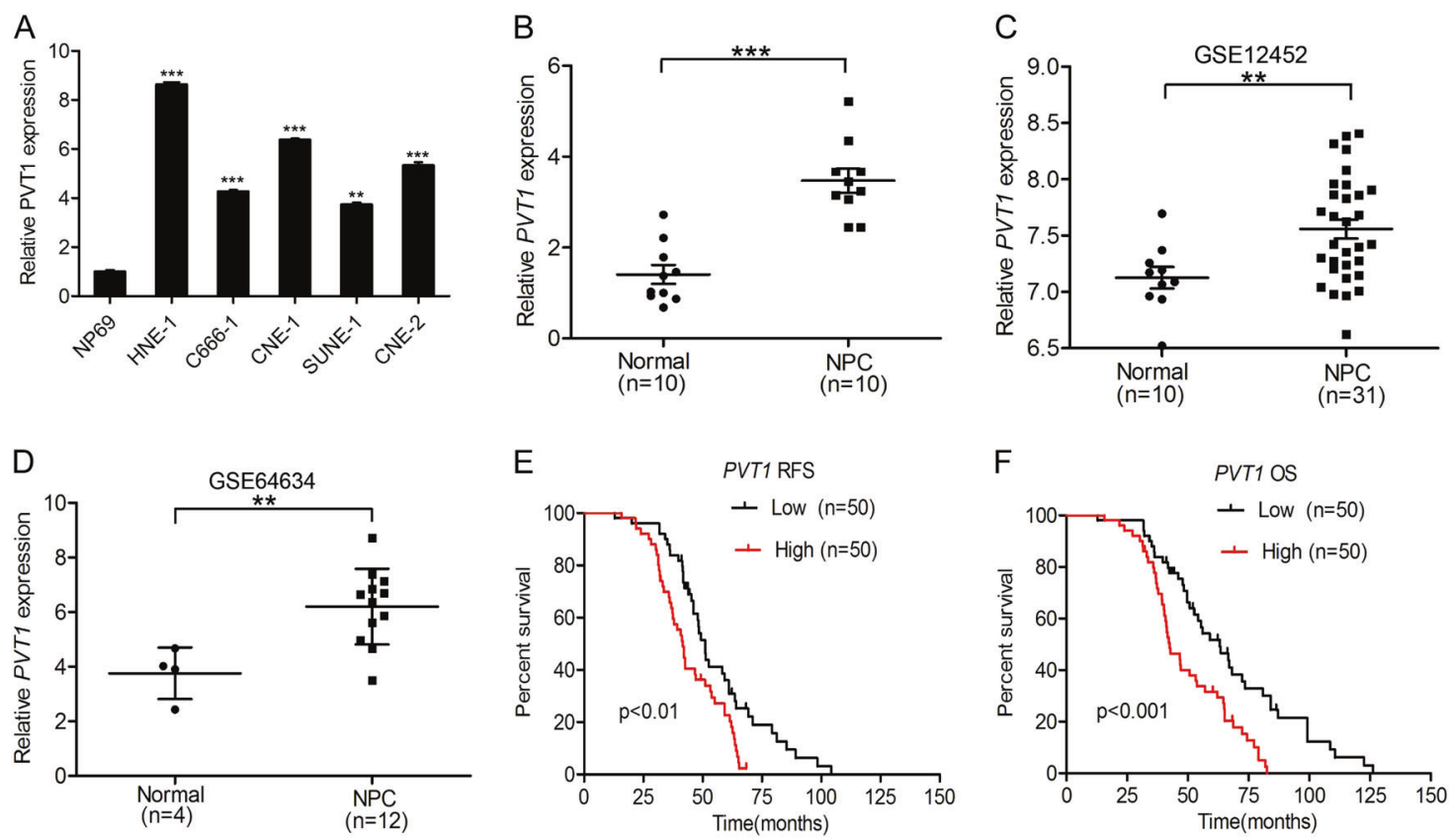

Fig. 1 The PVT1 lncRNA is upregulated in NPC and is associated with a poor prognosis in patients. a Expression of PVT1 mRNA is higher in NPC cell lines compared with the immortalized nasopharyngeal epithelial cell line. b Expression levels of PVT1 mRNA in ten clinical NPC tumors and ten freshly frozen normal nasopharyngeal specimens. c, d Expression levels of PVT1 mRNA in NPC tumors and normal nasopharyngeal specimens. PVT1 mRNA-expression data were obtained from the GSE12452 dataset [21] and the GSE64634 dataset [22] and analyzed. e, f, Kaplan-Meier analysis of relapse-free survival (RFS) and over survival (OS) for patients with high PVT1 mRNA-expression NPC versus low PVT1 mRNA-expression NPC. Error bars \pm SD. $* * P<0.01$. $* * * P<0.001$. Data are representative from three independent experiments 
was more highly expressed in NPC tissues relative to normal nasopharyngeal tissues (Fig. 1c, d). These results suggest that PVT1 may function as an oncogene that is involved in NPC progression.

Next, to assess clinical relevance of PVT1 expression in NPC, we examined the relationship between PVT1 expression and NPC patient survival via a Kaplan-Meier survival analysis. As shown in Fig. 1e, f, patients who exhibited high PVT1 expression had a shorter relapse-free survival and over survival. This indicates that PVT1 is closely associated with a poor prognosis in NPC patients. Moreover, we demonstrated that PVT1 is abundant within both the nucleus and cytoplasm of NPC (Supplementary
Fig. 1A, B). Taken together, these results indicate that PVT1 is upregulated in NPC and is associated with a poor prognosis in NPC patients.

\section{Knockdown of PVT1 disrupts NPC cell proliferation and tumor growth}

To determine the functions of PVT1 in NPC, we first constructed HNE-1 cells expressing a PVT1-specific shRNA (shPVT1) or a nonsilencing control shRNA (shC), and then performed RNA sequencing to compare their gene expression profiles. As shown in Fig. 2a, 313 genes were upregulated and 137 genes were downregulated ( $\geq 1.5$-fold,

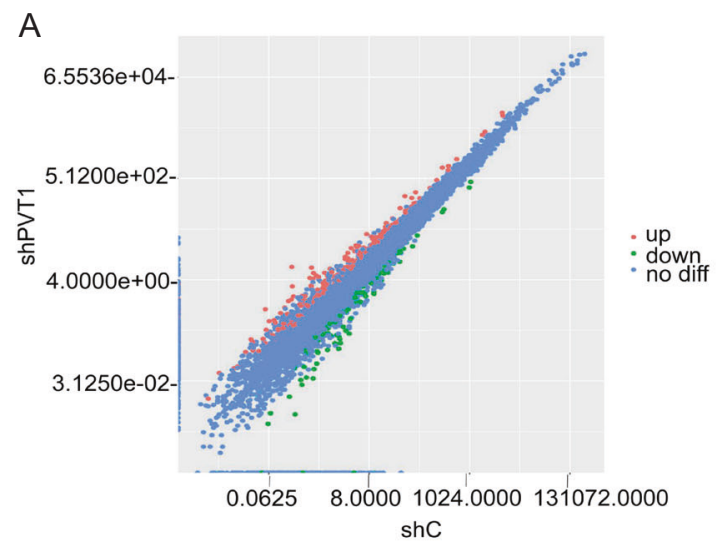

B
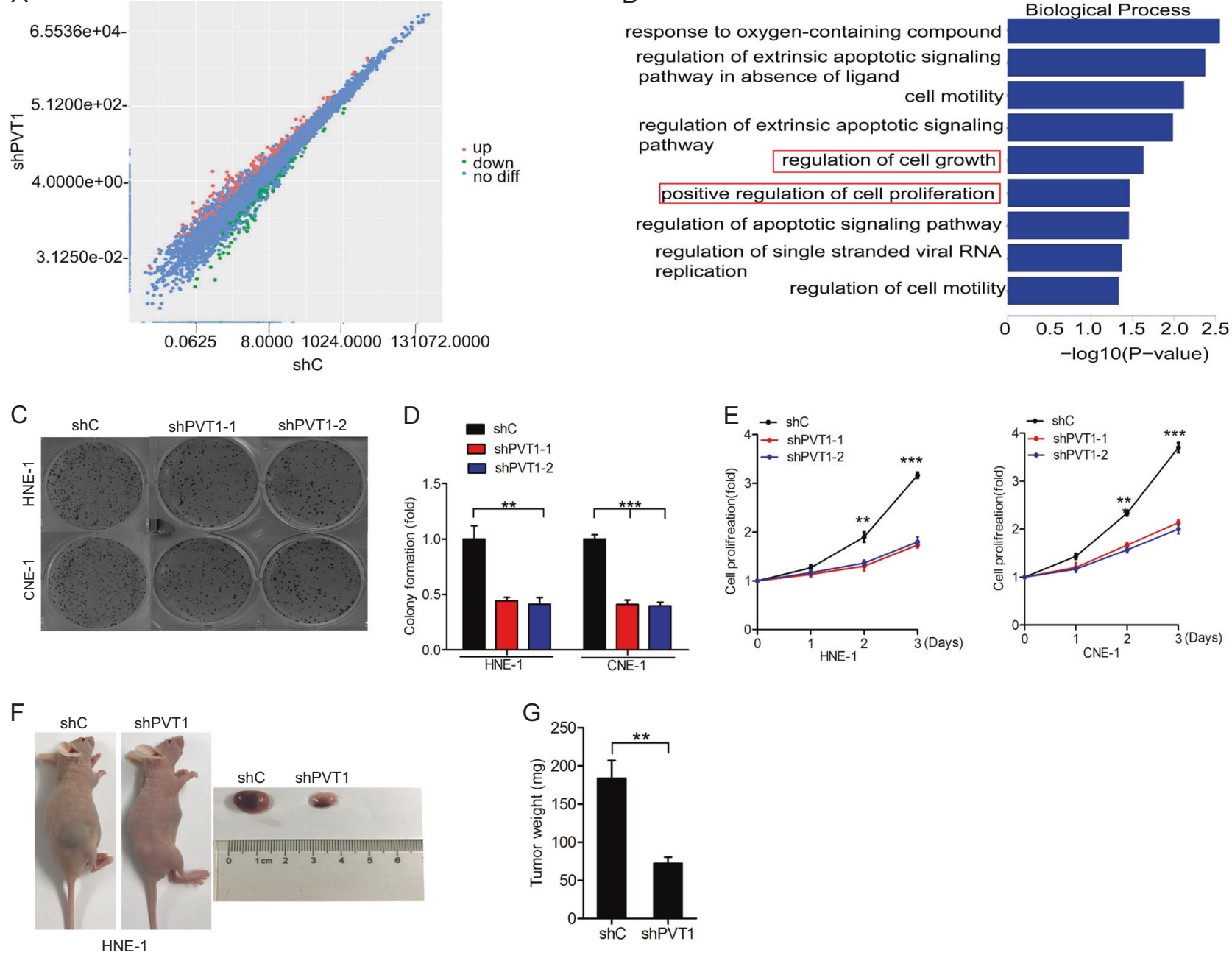

Fig. 2 Knockdown of PVT1 disrupts NPC cell proliferation and tumor growth. a Scatter plot of 16764 gene expression in HNE-1 cells transfected with PVT1 shRNA ( $y$-axis) compared with the control group ( $x$-axis). Green dots, set of genes exhibiting significant downregulation upon PVT1 knockdown; red dots, set of genes exhibiting significant upregulation upon PVT1 knockdown; black dots, the genes without significant change. b The biological process signaling pathways response upon PVT1 knockdown by Gene GO analyses. c Effects of PVT1 knockdown on NPC cell colony formation. d

Quantification of colony formation in c. e Effects of PVT1 knockdown on NPC cell proliferation. $f$ Representative images of PVT1 knockdown-inhibited HNE-1 subcutaneous tumor generation. Tumors were harvested at 3-4 weeks after implantation. Data were from two independent experiments with five mice per group with similar results. g Quantification of tumor weight in f. Error bars \pm SD. $* * P<0.01$. $* * * P<0.001$. Data are representative from three independent experiments 
$p<0.05)$ in HNE-1 cells in which PVT1 was knocked down.

A gene ontology (GO) analysis was then performed in order to investigate the pathways responsive to PVT1 knockdown. Our results indicated that these differentially expressed genes were enriched in genes linked with proliferation (Fig. 2b). These results suggest that PVT1 may be a vital regulatory lncRNA involved in regulating NPC cell proliferation.

To provide functional confirmation of the above findings, we next revealed that PVT1 knockdown (Supplementary Fig. 2) inhibits colony formation (Fig. 2c, d) and cell proliferation in HNE-1 and CNE-1 cells (Fig. 2e). We next further sought to elucidate the biological role of PVT1 in NPC tumorigenesis, and found that PVT1 knockdown significantly inhibited NPC tumor growth in mice in the PVT1 silenced group relative to the control group (Fig. 2f, g). These results thus confirm that PVT1 plays a role in regulating the proliferation and growth of NPC tumors.

\section{PVT1 promotes NPC cell proliferation via HIF-1a}

To explore the mechanisms by which PVT1 regulates cell proliferation, we performed Kyoto Encyclopedia of Genes and Genomes (KGEE) to analyze the downstream pathways affected by PVT1 expression, revealing that PVT1 expression is positively correlated with the HIF-1 signaling pathway (Fig. 3a). Moreover, HIF-1 $\alpha$, which is a key mediator of the HIF-1 signaling pathway, mRNA (Fig. 3b) and protein levels (Fig. 3c, d) was downregulated upon PVT1 knockdown. These results suggest that HIF-1 $\alpha$ may play a vital role in the PVT1 regulatory network.

Next, to determine whether PVT1 mediates HIF-1 $\alpha$ mRNA stability, we treated HNE-1 cells with the RNA synthesis inhibitor Actinomycin D for 0,1 , or $2 \mathrm{~h}$. Relative to the control group, we found that HIF-1 $\alpha$ mRNA was degraded more quickly in the PVT1 depletion group (Fig. 3e), thus suggesting that PVT1 regulates HIF-1 $\alpha$ mRNA stability.

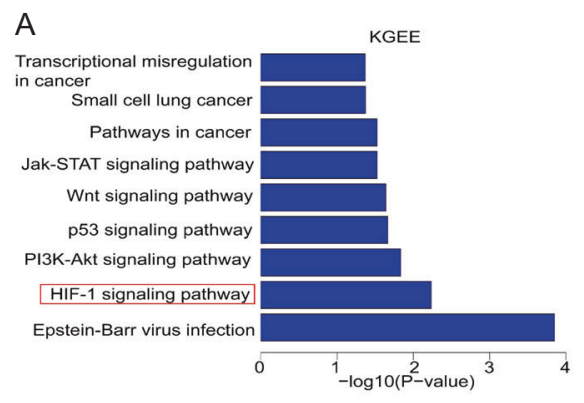

B

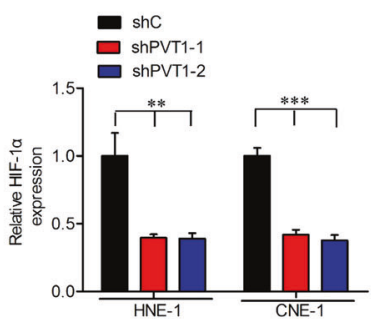

C

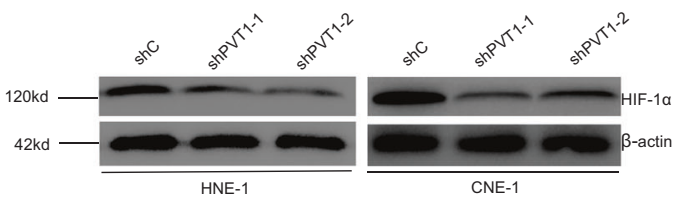

D

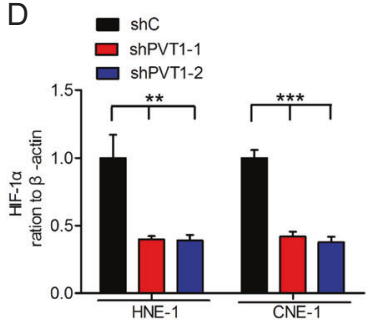

G

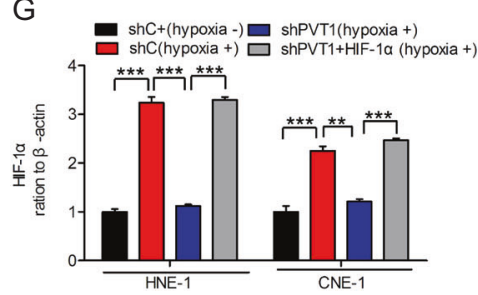

Fig. 3 PVT1 promotes NPC cell proliferation via HIF-1 $\alpha$. a The signaling pathways response upon PVT1 knockdown by Gene KGEE analyses. Quantitative RT-PCR (b) and WB (c) analyses of effects of PVT1 knockdown on HIF- $1 \alpha$ activation. d Quantification of HIF- $1 \alpha$ protein in c. e Reduction of HIF-1 $\alpha$ mRNA stability in PVT1 knockdown HNE-1 cells compared with control cells. Cells were treated with $1 \mu \mathrm{g} / \mathrm{ml}$ actinomycin D and RNA was extracted at the time of 0,1 , and $2 \mathrm{~h}$. f HIF- $1 \alpha$ protein expression assays for HNE- 1 and CNE-1 under hypoxia conditions $\left(1 \% \mathrm{O}_{2}\right)$. g Quantification of HIF-1 $\alpha$

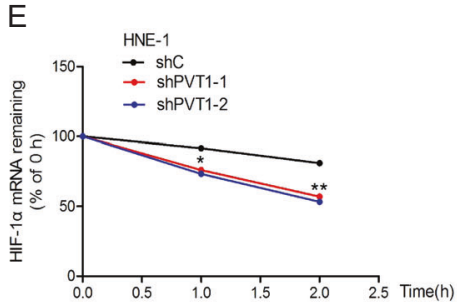

$\mathrm{F}$

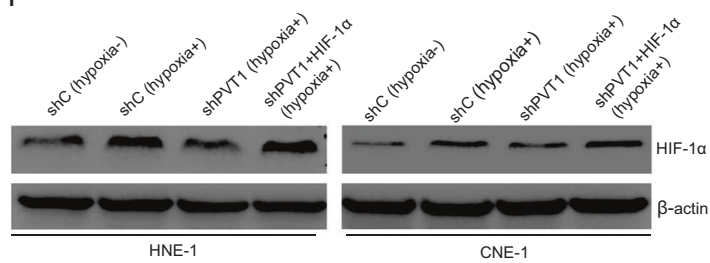

$\mathrm{H}$

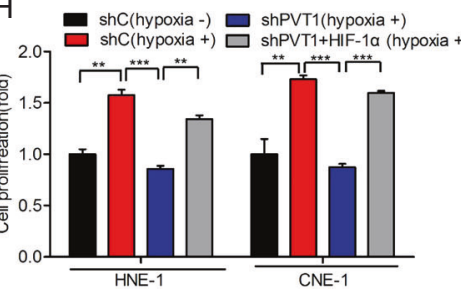

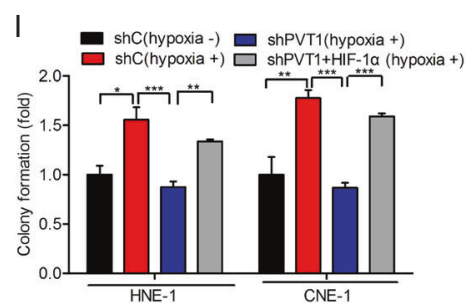

protein in $\mathbf{f}$. Cell proliferation (h) and colony formation (i) assays for HNE-1 cells under hypoxic conditions. Hypoxia induced the cell proliferation and colony formation of NPC cells. The cell proliferation and colony formation abilities of NPC cells under hypoxic conditions were suppressed by PVT1 knockdown, and these suppressed cell proliferation and colony formation abilities of NPC cells were restored by HIF- $1 \alpha$ overexpression. Error bars \pm SD. $* P<0.05$. $* * P<0.01$. $* * * P<0.001$. Data are representative from three independent experiments 
To assess whether PVT1-mediated HIF-1 $\alpha$ expression contributes to NPC cell proliferation, we overexpressed HIF-1 $\alpha$ in PVT1-knockdown HNE-1 cells (Fig. 3f-g), and found that this restored the proliferation of these cells (Fig. 3h) and their colony formation abilities (Fig. 3i). These results further suggested that PVT1 thus regulates cell proliferation at least in part via modulating $\mathrm{HIF}-1 \alpha$ stability.

\section{PVT1 binds to KAT2A in NPC cells}

Recent studies have reported that IncRNAs can function as sponges to bind histone acetyltransferases and thereby modulate their downstream gene expression [5, 23]. We hypothesized that PVT1-mediated tumorigenesis may therefore depend upon its ability to bind certain histone acetyltransferases. We first found that three histone acetyltransferases (KAT2A, KAT2B, and KAT8) were markedly upregulated and two (KAT5 and KAT6A) were markedly downregulated in three freshly frozen normal nasopharyngeal specimens relative to three clinical NPC tumors via RNA sequencing ( $\geq 1.5$-fold, $p<0.05$ ) (Supplementary Fig. 3A). Next, we found that PVT1 bound to KAT2, but not to the other histone acetyltransferases tested (Fig. 4a and Supplementary Fig. 3B). Previous reports indicate that WDR5 and KAT2A can form a complex and bind to the lncRNA GClnc1 in order to promote gastric carcinogenesis [5]. Consistent with this mechanism, we found that the histone methyltransferase WDR5 was also clearly upregulated in NPC tissues relative to in normal nasopharyngeal tissues, and we further confirmed that PVT1 bound to WDR5 (Supplementary Fig. 3B). Moreover, we validated that both KAT2A and WDR5 protein expression levels were higher in NPC tissues compared with that in normal nasopharyngeal tissues (Supplementary Fig. 3C, D), but PVT1 knockdown had no effect on KAT2A or WDR5 expression (Supplementary Fig. 3E, F). These
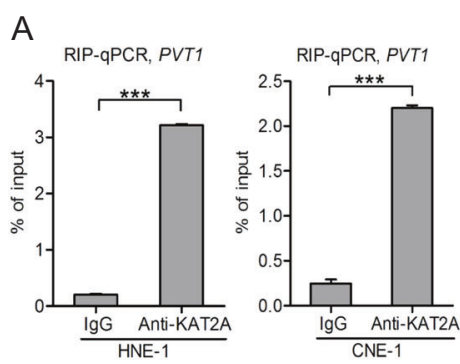

B

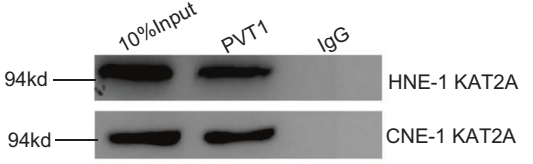

$1: 227-788 \mathrm{bp} ; \Delta 2: 1-166 \mathrm{bp}$ $\Delta 3: 1768-1957 \mathrm{bp} ; \Delta 4: 1407-1732 \mathrm{bp}$ $\Delta 5: 899-1374$ bp

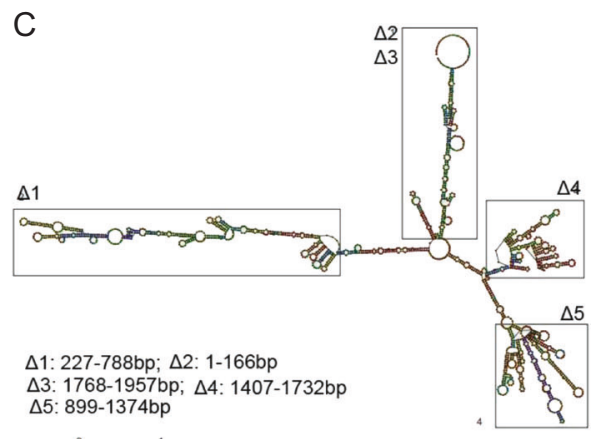

E
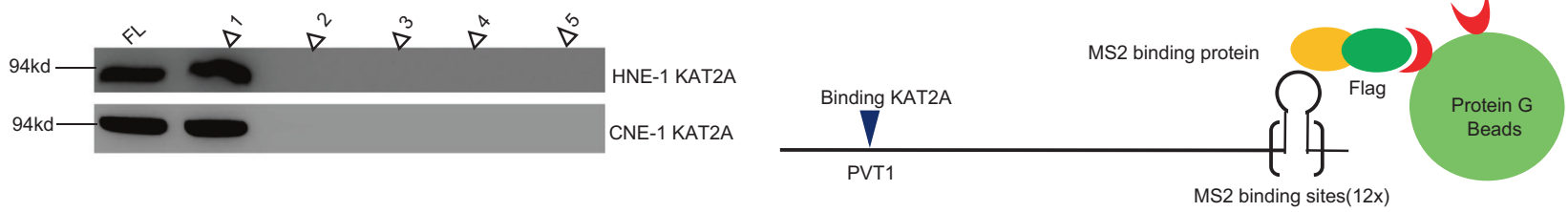

$\mathrm{F}$

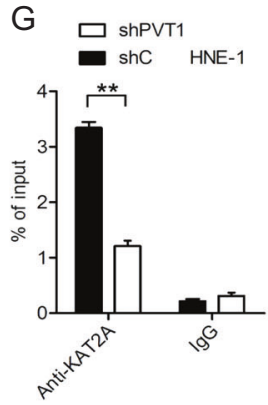

Fig. 4 PVT1 binds to KAT2A in NPC cells. a The RIP-qPCR assay of the binding of KAT2A with PVT1 in HNE-1 and CNE-1 cells. b Biotinylated PVT1 was incubated with extracts (HNE-1 and CNE-1 cells), treated with streptavidin beads, and binding proteins were resolved in a gel. The WB assay of the specific binding of KAT2A and PVT1. c Graphic illustration of predicted PVT1 secondary structure (http://www.lncipedia.org), and the truncation of PVT1 in accordance with the stem-loop structure. d Secondary structure regions of PVT1 were treated as in $\mathbf{b}$, and binding KAT2A was detected by the WB assay. e, f, WB detection of KAT2A binding upon secondary structure regions of PVT1 after Flag-MS2bp-MS2bs-based pull-down assay. g Effects of PVT1 knockdown on the specific binding of KAT2A and PVT1. Error bars \pm SD. $* * P<0.01$. $* * * P<0.001$. Data are representative from two independent experiments 
results thus suggest that PVT1 may act as a scaffold for the WDR5 and KAT2A complexes in order to specify a pattern of histone modification. We next utilized a biotin-avidin pulldown system to detect the association between PVT1 and KAT2A (Fig. 4b), and used deletion-mapping system (Fig. 4c, d) to detect whether KAT2A binds to with a specific region of PVT1. This approach identified a 227-788 nt region at the $5^{\prime}$ end of PVT1 required for its binding to KAT2A (Fig. 4d).

Finally, to further confirm that PVT1 binds to KAT2A, we used a Flag-MS2bp-MS2bs system to perform an RNA pulldown experiment (Fig. 4e). As shown in Fig. 4f, we found that KAT2A also bound to a 227-788 nt region at the $5^{\prime}$ end of PVT1 in HNE-1 cells. RIP quantitative real-time PCR demonstrated that PVT1 depletion inhibited the binding between PVT1 and KAT2A (Fig. 4g). Taken together, these results suggest that KAT2A binds to PVT1 in NPC cells.

\section{PVT1 stabilizes HIF-1a via KAT2A}

As HIF- $1 \alpha$ is a critical downstream effector of PVT1 in NPC cells, we next assessed whether PVT1 stabilizes
HIF- $1 \alpha$ via interacting with KAT2A in these cells. As shown in Fig. 5a-c, PVT1 depletion decreased HIF-1 $\alpha$ protein and mRNA in HNE-1 and CNE-1 cells. However, overexpression of KAT2A rescued PVT1 knockdownmediated loss of HIF-1 $\alpha$ protein expression (Fig. 5d, e), mRNA expression (Fig. 5f), and mRNA stability (Fig. 5g). Moreover, overexpression of KAT2A restored PVT1 depletion-mediated loss of cell proliferation (Fig. 5h) and colony formation (Fig. 5i) in HNE cells. Taken together, these data show that the IncRNA PVT1 stabilizes HIF- $1 \alpha$ via KAT2A.

\section{KAT2A acetyltransferase activity is required for PVT1-driven HIF-1a stabilization}

To explore the potential mechanisms by which PVT1 regulates HIF-1 $\alpha$ stabilization through KAT2A, we next assessed histone $\mathrm{H} 3$ acetylation expression levels via western blotting. H3K9 has been reported to be a target of KAT2A [5]. As shown in Fig. 6a and Supplementary Fig. 4A, PVT1 knockdown decreased H3K9 acetylation levels, but not KAT2A expression in HNE-1 and CNE-1

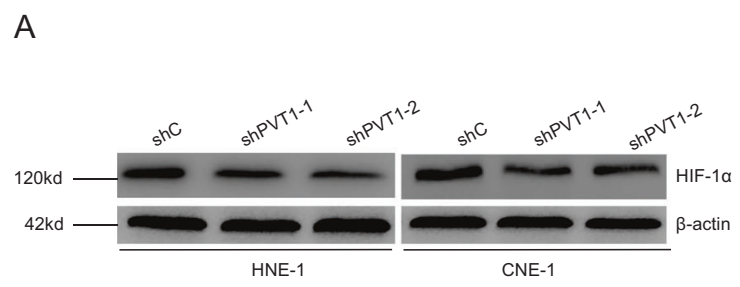

B
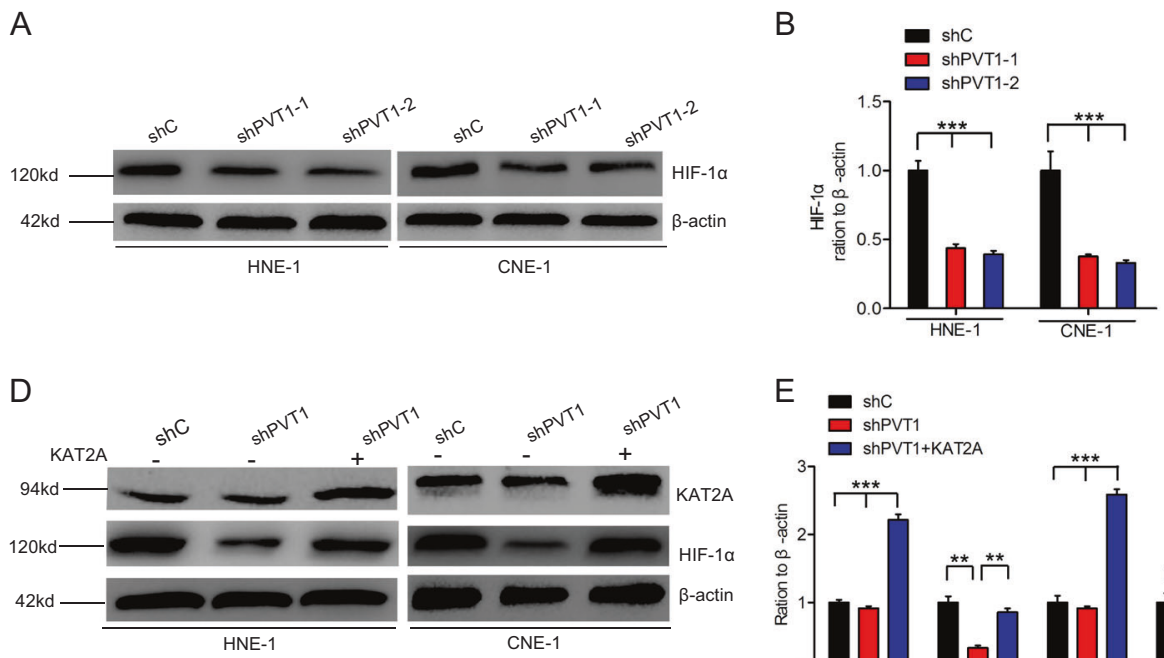

$\mathrm{E}$

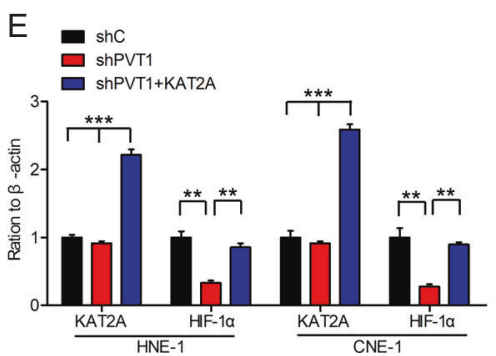

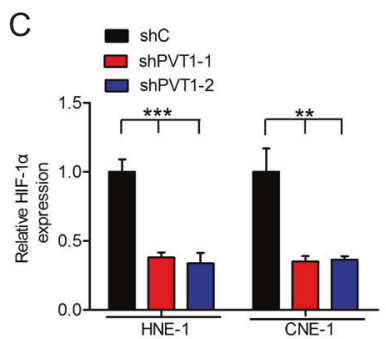

$\mathrm{F}$

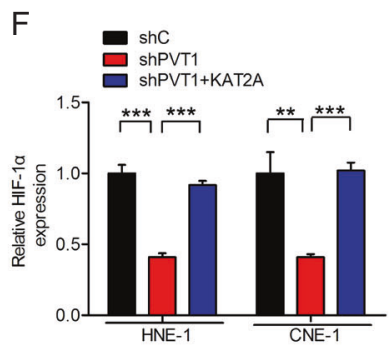

G

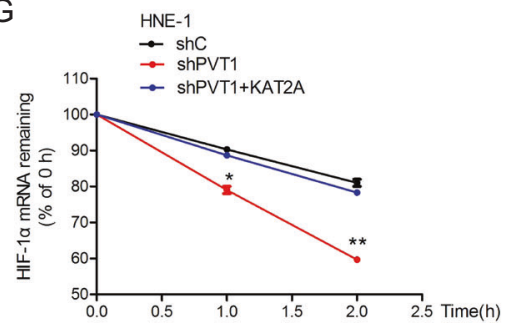

$\mathrm{H}$

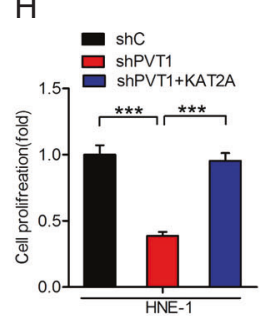

I

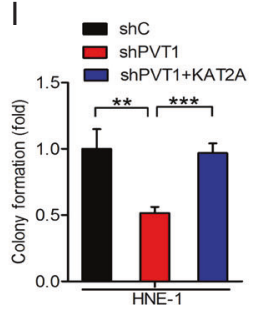

Fig. 5 PVT1 stabilizes HIF-1 $\alpha$ via KAT2A. a Effects of PVT1 knockdown on HIF-1 $\alpha$ protein in HNE-1 and CNE-1 NPC cells. b Quantification of HIF-1 $\alpha$ protein in a. c Effects of PVT1 knockdown on HIF- $1 \alpha$ mRNA in HNE- 1 and CNE-1 NPC cells. $\mathbf{d}$ Overexpression of KAT2A restores PVT1 knockdown-inhibited HIF-1 $\alpha$ protein. e Quantification of HIF- $1 \alpha$ and KAT2A proteins in d. Overexpression of KAT2A restores PVT1 knockdown-inhibited HIF-1 $\alpha$ mRNA (f), HIF- $1 \alpha$ mRNA stability (g), NPC cell proliferation (h), and colony formation (i). Error bars \pm SD. $* * P<0.01$. $* * * P<0.001$. Data are representative from three independent experiments 
A

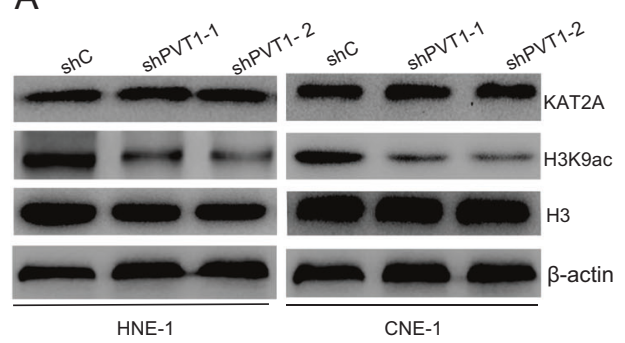

B

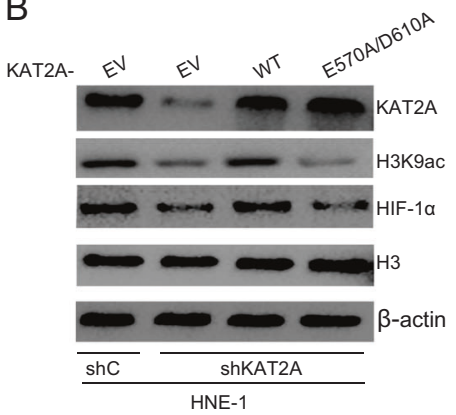

C

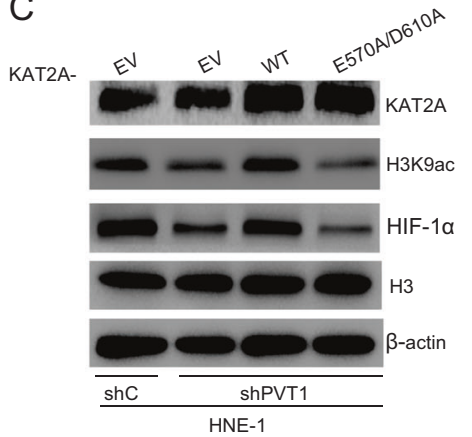

D

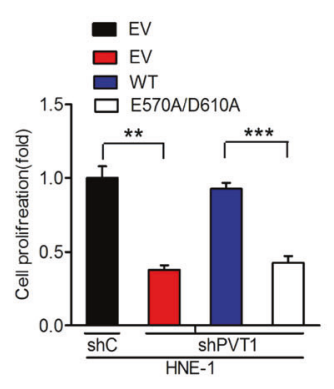

G

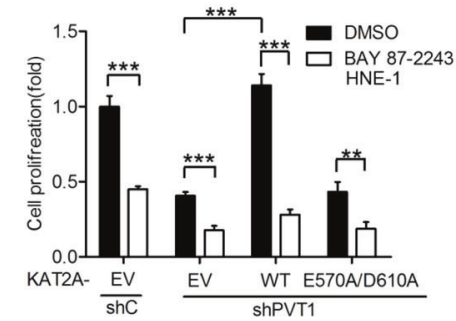

$\mathrm{E}$

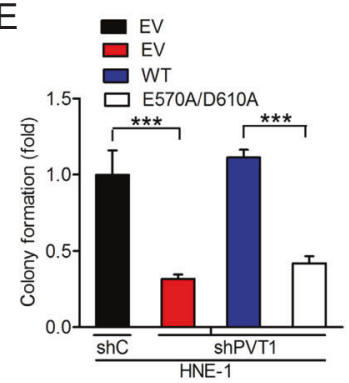

$\mathrm{H}$

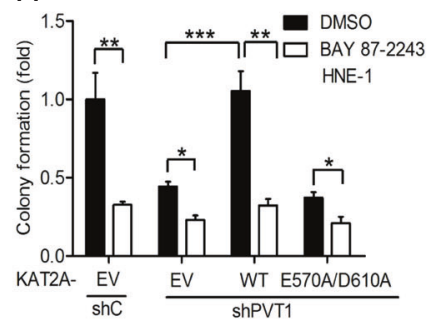

$\mathrm{F}$

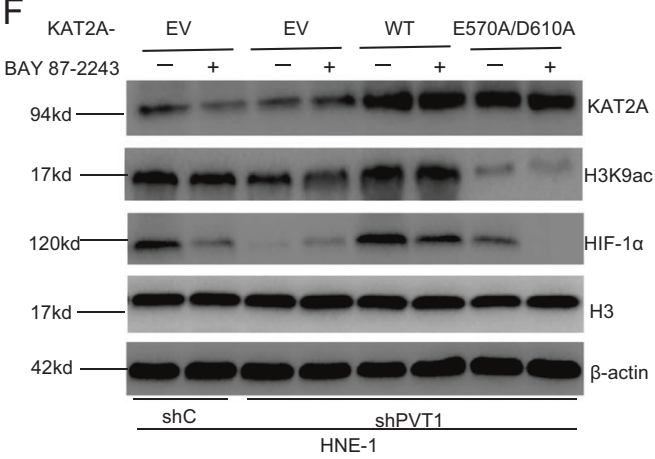

Fig. 6 KAT2A acetyltransferase activity is required for PVT1-driven HIF-1 $\alpha$ stabilization. a Effects of PVT1 knockdown on KAT2A and the acetylation of $\mathrm{H} 3 \mathrm{~K} 9$ expression in HNE-1 and CNE-1 NPC cells. $\beta$-actin and Histone $\mathrm{H} 3$ were used as controls. b Effects of overexpression of KAT2A wild-type and acetyltransferase activitydeficient mutant, E570A/D610A, on KAT2A depletion-inhibited HIF-1 $\alpha$ expression. c Overexpression of KAT2A wild type but not acetyltransferase activity-deficient mutant, E570A/D610A, restored
PVT1 depletion-inhibited HIF-1 $\alpha$ expression. Effects of overexpression of KAT2A wild-type and acetyltransferase activitydeficient mutant, E570A/D610A, on NPC cell proliferation (d) and colony formation (e). Inhibition of HIF- $1 \alpha$ reduced KAT2A-induced HIF- $1 \alpha$ protein expression (f), NPC cell proliferation (g), and colony formation (h). Error bars \pm SD. $* * P<0.01, * * * P<0.001$. Data are representative from three independent experiments

Fig. 4B). Moreover, as shown in Fig. 6c-e and Supplementary Fig. 4C, overexpression of KAT2 $\mathrm{A}^{\mathrm{WT}}$, but not KAT2A ${ }^{\text {E570A/D601A }}$, in HNE-1 NPC cells rescued PVT1 knockdown-mediated loss of HIF-1 $\alpha$ expression, cell proliferation, and colony formation. This indicates that KAT2A acetyltransferase activity is important for PVT1-mediated HIF-1 $\alpha$ stabilization.

Recent evidences have demonstrated that the lead structure BAY 87-2243 suppressed HIF-1 $\alpha$ protein levels and reduced HIF-1 target gene expression [25, 26]. Then, we investigated whether treatment with the HIF-1 $\alpha$ inhibitor BAY 87-2243 suppresses PVT1-mediated cell proliferation. As shown in Fig. 6f and Supplementary Fig. 4D, BAY 87-2243 treatment markedly suppressed HIF-1 $\alpha$ expression in HNE-1 cells transfected the KAT2A WT relative to E570A/D601A 
Isoform or emptor vector (EV) controls, whereas H3K9ac levels were unaffected by BAY 87-2243 treatment. We next assessed the effects of BAY 87-2243 on PVT1-mediated cell proliferation and colony formation, and found that this inhibitor markedly disrupted NPC cell proliferation and colony formation in KAT2A ${ }^{\text {WT }}$, but not KAT2 $\mathrm{A}^{\mathrm{E} 570 \mathrm{~A} / \mathrm{D} 601 \mathrm{~A}}$, overexpressing NPC cells relative to EV controls (Fig. 6g, h). These data demonstrate that KAT2A acetyltransferase activity is therefore required for PVT1-driven HIF-1 $\alpha$ stabilization.

\section{PVT1 promotes HIF-1a stability via KAT2A-mediated regulation of the TIF1 $\beta / \mathrm{H} 3 \mathrm{~K} 9 \mathrm{ac}$ complex}

Recently, the transcription intermediary factor (TIF) family of genes have been reported to bind to $\mathrm{H} 3$ acetylation sites in the genome and thereby regulate the expression of proximal genes in many cancers [27-29]. We hypothesized that PVT1 may promote KAT2A-mediated HIF- $1 \alpha$ expression via promoting $\mathrm{H} 3 \mathrm{~K} 9$ acetylation and thereby enhancing the binding of TIF genes and H3K9ac. This would in turn lead TIF family genes to function as transcriptional activators which promote downstream gene transcription, leading to HIF- $1 \alpha$ stability and thereby enhancing NPC tumorigenesis. To verify this hypothesis, we performed immunoprecipitation assays in NPC cells. As shown in Supplementary Fig. 5A, we found that H3K9ac bound to TIF1 $\alpha$ and TIF1 $\beta$, but not to TIF1 $\gamma$, NCOA1, NCOA2, or NCOA3. Next, we determined that overexpression of TIF1 $\beta$, but not of TIF1 $\alpha$, rescued PVT1 knockdown-mediated loss of HIF- $1 \alpha$ mRNA stability (Supplementary Fig. 5B, C). We therefore hypothesized that PVT1 promotes HIF-1 $\alpha$ mRNA stability via a TIF1 $\beta / H 3 K 9 a c$ complex. We found that knockdown of PVT1 significantly attenuated the binding of TIF1 $\beta$ and H3K9ac, whereas overexpression of KAT2A rescued this defect (Fig. $7 \mathrm{a}-\mathrm{c}$ and Supplementary 6A-C). Next, to further assess which domain of TIF $1 \beta$ regulates its binding to $\mathrm{H} 3 \mathrm{~K} 9 \mathrm{ac}$, we employed a deletion-mapping approach (Fig. 7d), and then transfected these deletion mutant protein isoforms into HNE-1 cells.
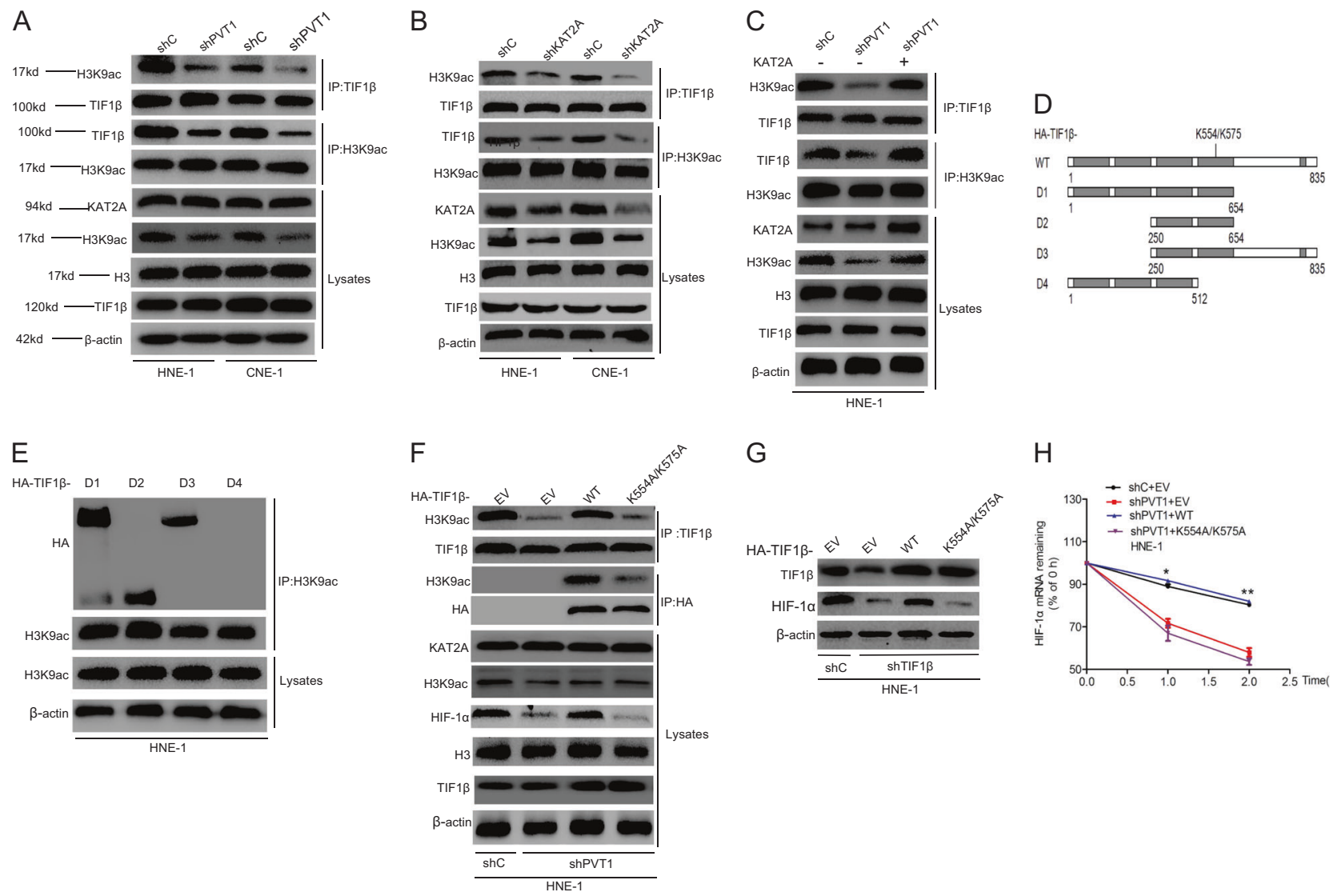

G

$\mathrm{H}$
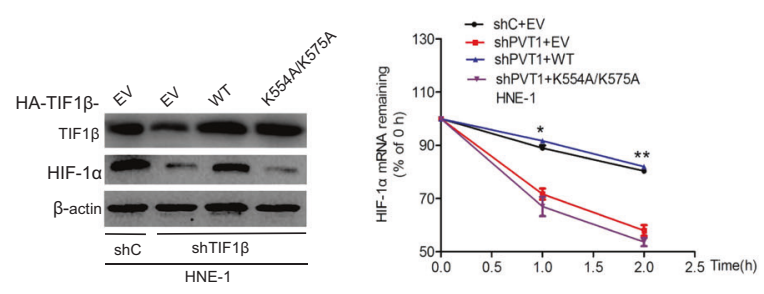

Fig. 7 PVT1 promotes HIF-1 $\alpha$ stability via KAT2A -mediated regulation of the TIF $1 \beta / \mathrm{H} 3 \mathrm{~K} 9 \mathrm{ac}$ complex. a Immunoprecipitation and WB analyses of effects of PVT1 knockdown on H3K9ac association with TIF1 $\beta$. b KAT2A knockdown inhibited the association of H3K9ac with TIF1 $\beta$. c Overexpression of KAT2A rescues PVT1 knockdown-inhibited the binding of H3K9ac with TIF1 $\beta$. d Schematics of TIF $1 \beta^{\mathrm{WT}}$ and various TIF1 $\beta$ deletion mutants. e H3K9ac binds with TIF1 $\beta$ with amino acid residues 512-654. f Overexpression

of TIF1 $\beta$ wild type but not K554A/K575A mutant restores PVT1 knockdown-inhibited the binding of H3K9ac with TIF1 $\beta$. g Overexpression of TIF1 $\beta$ wild type but not K554A/K575A mutant restores TIF1 $\beta$ knockdown-inhibited HIF-1 $\alpha$ expression. $\mathbf{h}$ Overexpression of TIF1 $\beta$ wild type but not K554A/K575A mutant restores PVT1 knockdown-inhibited HIF-1 $\alpha$ mRNA stability. Error bars \pm SD. $* P<$ 0.05 . $* * P<0.01$. Data are representative from three independent experiments 
We found that the D1, D2, and D3 mutants, but not the D4 mutant, were able to bind to H3K9ac (Fig. 7e), suggesting that amino acids 512-654 of TIF1 $\beta$ are required for binding to H3K9ac. To further confirm that TIF1 $\beta$ is important for PVT1-mediated HIF-1 $\alpha$ stabilization, we overexpressed either HA-tagged TIF1 $\beta$ WT or a K554A/K575A TIF1 $\beta$ mutant (K554A/K575A) in which the binding of TIF $1 \beta$ and H3K9ac was predicted to be disrupted by The Eukaryotic Linear Motif Resource for Functional Sites of Proteins (http://elm.eu.org). As shown in Fig. 7f and Supplementary Fig. 6D, overexpression of HA-TIF1 $\beta$ WT, but not HA-TIF1 $\beta-K 554 \mathrm{~A} /$ K575A mutant, could restored PVT1 depletion-mediated loss of HIF-1 $\alpha$ expression instead of H3K9 acetylation. Moreover, overexpression of HA-TIF1 $\beta$ WT was able to rescue PVT1 depletion-mediated loss of binding between TIF1 $\beta$ and H3K9ac, but overexpression of the HA-TIF1 $\beta-K 554 \mathrm{~A} /$ K575A mutant did not affect this interaction. Exogenous expression of TIF1 $\beta$ WT could restored TIF1 $\beta$ depletionmediated loss of HIF- $1 \alpha$ expression, but overexpression of the HA-TIF1 $\beta-K 554 A / K 575 A$ mutant had no effects (Fig. 7g and Supplementary Fig. 6E). Consistent with these findings, overexpression of HA-TIF1 $\beta$ WT restored PVT1 depletionmediated loss of cell proliferation, colony formation, and HIF$1 \alpha$ mRNA stability, while overexpression of Flag-TIF1 $\beta$ K554A/K575A mutant did not alter these characteristics (Fig. 7h, Supplementary Fig. 6F, G). Taken together, these results demonstrate that PVT1 promotes HIF- $1 \alpha$ stability through a KAT2A-mediated TIF1//H3K9ac complex.

\section{PVT1 promotes TIF1 $\beta /$ H3K9ac complex-mediated NF90 transcriptional activation to regulate HIF-1a stability}

As NF90 is known to stabilize HIF-1 $\alpha$ and to regulate its gene expression [30], we further hypothesized that PVT1 may promote HIF- $1 \alpha$ mRNA stability via TIF $1 \beta / \mathrm{H} 3 \mathrm{~K} 9 \mathrm{ac}$ complex-mediated regulation of NF90 transcription. Consistent with this, we found that knockdown of PVT1 suppressed NF90 protein (Fig. 8a and Supplementary Fig. 7A) and mRNA expression levels (Fig. 8b). However, overexpression of KAT2A restored PVT1 depletionmediated loss of NF90 expression (Fig. 8c and Supplementary Fig. 7B). Furthermore, we performed ChIP assays to determine that KAT2A and WDR5 bind to the NF90 promoter at the -945 to -621 locus (Fig. 8d, e). NF90 knockdown-inhibited HIF-1 $\alpha$ protein (Fig. 8f and Supplementary Fig. 7C) and HIF-1 $\alpha$ mRNA (Fig. 8g). We further found that overexpression of NF90 restored PVT1 or KAT2A depletion-mediated loss of HIF- $1 \alpha$ expression (Fig. 8h-i and Supplementary Fig. 7D, E). Interestingly, overexpression of HA-TIF1 $\beta$ WT rescued PVT1 knockdown-mediated loss of NF90 and HIF-1 $\alpha$, and the binding between the NF90 promoter and the TIF1 $\beta / \mathrm{H} 3 \mathrm{~K} 9 \mathrm{ac}$ complex (Fig. 8j-k and Supplementary Fig. 7F), while overexpression of HA-TIF1 $\beta$ WT combined with KAT2A knockdown or binding mutant isoforms of the TIF1 $\beta$ / H3K9ac complex alone did not affect it (Fig. 8j-k and Supplementary Fig. 7F). These data thus show that PVT1 promotes TIF1//H3K9ac complex-mediated NF90 transcription as a means of regulating HIF- $1 \alpha$ stability.

\section{PVT1 suppresses the radiosensitivity of NPC cell lines by increasing the interaction between TIF1 $\beta$ and H3K9ac}

As radiation is a primary treatment for NPC, and PVT1 suppresses radiosensitivity in NPC [20], we reasoned that the molecular mechanism by which PVT1 regulates cancer progression may be closely related to NPC radiosensitivity. We found that PVT1 overexpression significantly increased the number of colonies formed when cells were treated via radiation relative to untreated controls in a dose-dependent manner (Fig. 9a, b), whereas PVT1 knockdown showed the opposite effects (Fig. 9c). Importantly, we found that PVT1 overexpression significantly increased cell proliferation and PVT1 knockdown markedly decreased cell proliferation after exposure to $4 \mathrm{~Gy}$ radiation (Fig. 9d, e). Consistent with this, tumor growth in vivo also recapitulated these findings (Fig. 9f-i).

To investigate how PVT1 suppresses cell proliferation after radiation, we first examined that radiation had no effects on KAT2A, H3K9ac, or TIF1 $\beta$ protein expression, but did inhibit NF90 and HIF-1 $\alpha$ expression (Fig. 9j and Supplementary Fig. 8A). However, overexpression of PVT1 restored radiation-mediated loss of NF90 and HIF- $1 \alpha$ expression (Fig. 9j and Supplementary Fig. 8A). Furthermore, radiation did not affect the binding of PVT1, KAT2A, and WDR5 (Fig. 9k). We thus hypothesized that radiation disturbed the interaction between $\mathrm{H} 3 \mathrm{~K} 9 \mathrm{ac}$ and TIF1 $\beta$, thereby decreasing NF90 transcription. To test our hypothesis, we detected that radiation inhibited the interaction between $\mathrm{H} 3 \mathrm{~K} 9 \mathrm{ac}$ and $\mathrm{TIF} 1 \beta$, and thus suppressed H3K9ac and TIF1 $\beta$-mediated enhancement of NF90 transcription (Fig. 91, m and Supplementary Fig. 8B). In conclusion, these results show that PVT1 suppresses the radiosensitivity of NPC cell lines by disturbing the interaction between TIF1 $\beta$ and H3K9ac.

\section{Discussion}

In this study, we identify a new putative mechanism by which the lncRNA PVT1 serves as a scaffold for the chromatin modification factor KAT2A, and thereby stabilizes HIF- $1 \alpha$ via H3K9ac/TIF1 $\beta$ complex-mediated NF90 transcription in a manner critical for NPC tumorigenesis (Fig. 10). 
A

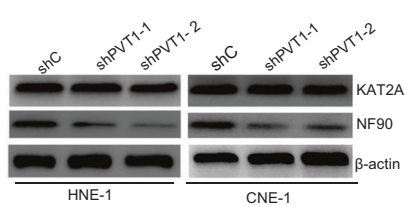

B

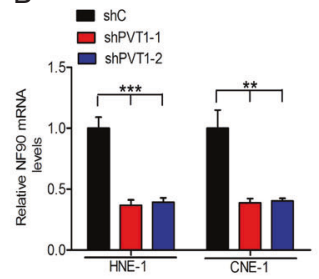

C

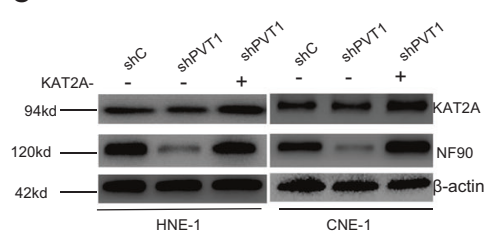

G

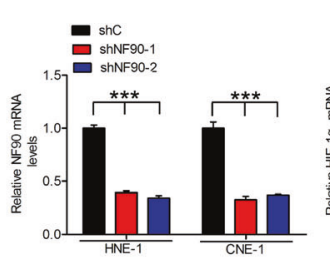

D KATZA CHP-APCR HNE-1

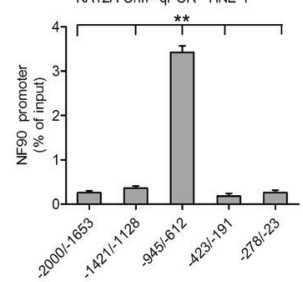

E

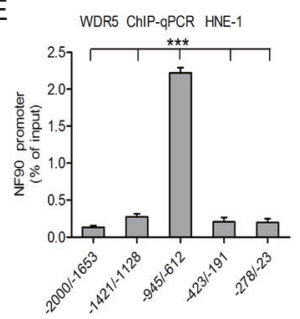

$\mathrm{F}$

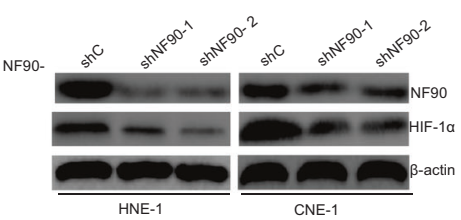

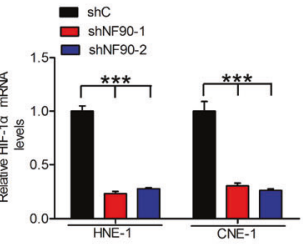

$\mathrm{H}$

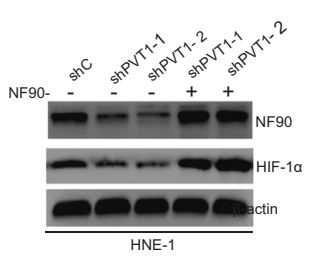

I

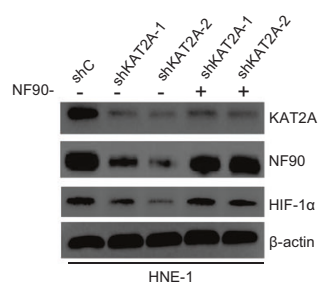

$J$

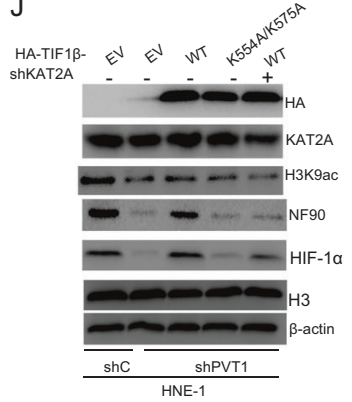

K

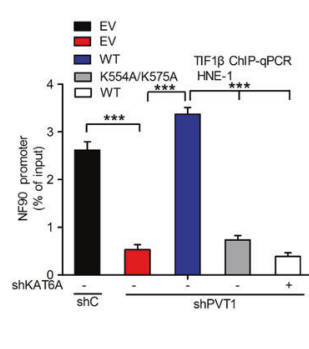

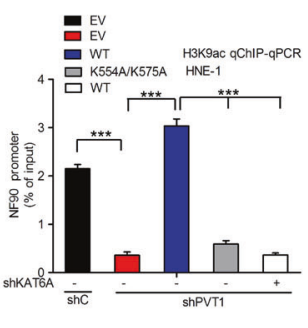

Fig. 8 PVT1 promotes TIF1ß/H3K9ac complex-mediated NF90 transcriptional activation to regulate HIF- $1 \alpha$ stability. Effects of PVT1 knockdown on NF90 protein (a) and mRNA (b) expression in HNE-1 and CNE-1 NPC cells. c Overexpression of KAT2A restored PVT1 knockdown-inhibited NF90 expression. d, e The ChIP-qPCR assay of the association of KAT2A or WDR5 with the NF90 promoter. After using antibodies against KAT2A, WDR5, or control IgG, qPCR assays were conducted using primers corresponding to five different loci of the NF90 promoter. Effects of NF90 knockdown on HIF-1 $\alpha$ protein (f) and mRNA (g) expression in HNE-1 and CNE-1 NPC cells.
Overexpression of NF90 restored PVT1 (h) and KAT2A (i) knockdown-inhibited NF90 expression. $\mathbf{j}$ Effects of overexpression of TIF1 $\beta$ wild-type and K554A/K575A mutant on PVT1 knockdowninhibited HIF-1 $\alpha$, NF90, and the acetylation of H3K9 expression in HNE-1 cells. $\mathbf{k}$ Representation of overexpression of TIF1 $\beta$ wild type but not K554A/K575A mutant on PVT1 knockdown-inhibited the association of KAT2A or WDR5 with the NF90 promoter. Error bars \pm SD. $* * P<0.01$. $* * * P<0.001$. Data are representative from three independent experiments
Our results reveal that PVT1 functions as an oncogene in the context of NPC tumorigenesis. Increasing evidences demonstrate that abnormal expression of many lncRNAs can play a critical role in malignant NPC progression [20, 30]. Recent PVT1 was found to promote cell proliferation via regulating the cell cycle in many cancers $[31,32]$. In this study, we found that expression of PVT1 was upregulated in NPC specimens. Moreover, PVT1 expression is negatively correlated with survival in NPC patients. PVT1 depletion via shRNA inhibited NPC cell proliferation and colony formation in vitro, and NPC tumorigenesis in vivo. These results therefore support a model in which PVT1 is critical for NPC tumorigenesis.

Our results also reveal that PVT1 mediates NPC cell proliferation via HIF-1 $\alpha$ stabilization. Although PVT1 contributed to the regulation of cell proliferation in cancer cells $[31,32]$, the mechanisms by which PVT1 regulates cell proliferation in NPC remained unclear. PVT1 knockdown predicts poor prognostic and induces radioresistance by mediate cell apoptosis and DNA repair in NPC [20]. PVT1 promotes colony formation via the PI3K/AKT signaling pathway in NPC [33]. Our KGEE analysis demonstrated that PVT1 expression was positively correlated with a hypoxia phenotype. This is consistent with recent clinical data showing that primary nasopharynx tumors are primarily hypoxic [34, 35], suggesting that a hypoxic environment may be crucial for NPC progression. Moreover, HIF-1 $\alpha$, a core mediator of the hypoxia response [36, 37], was downregulated after PVT1 depletion. In addition, HIF-1 $\alpha$ mRNA stability has also been reported to be regulated by lncRNAs and then affects NPC carcinogenesis [30, 38]. Our results also showed that the knockdown of PVT1 reduced HIF- $1 \alpha$ mRNA stability, which was restored by overexpression of HIF-1 $\alpha$. NF90, known as a double-stranded RNA-binding protein, is 

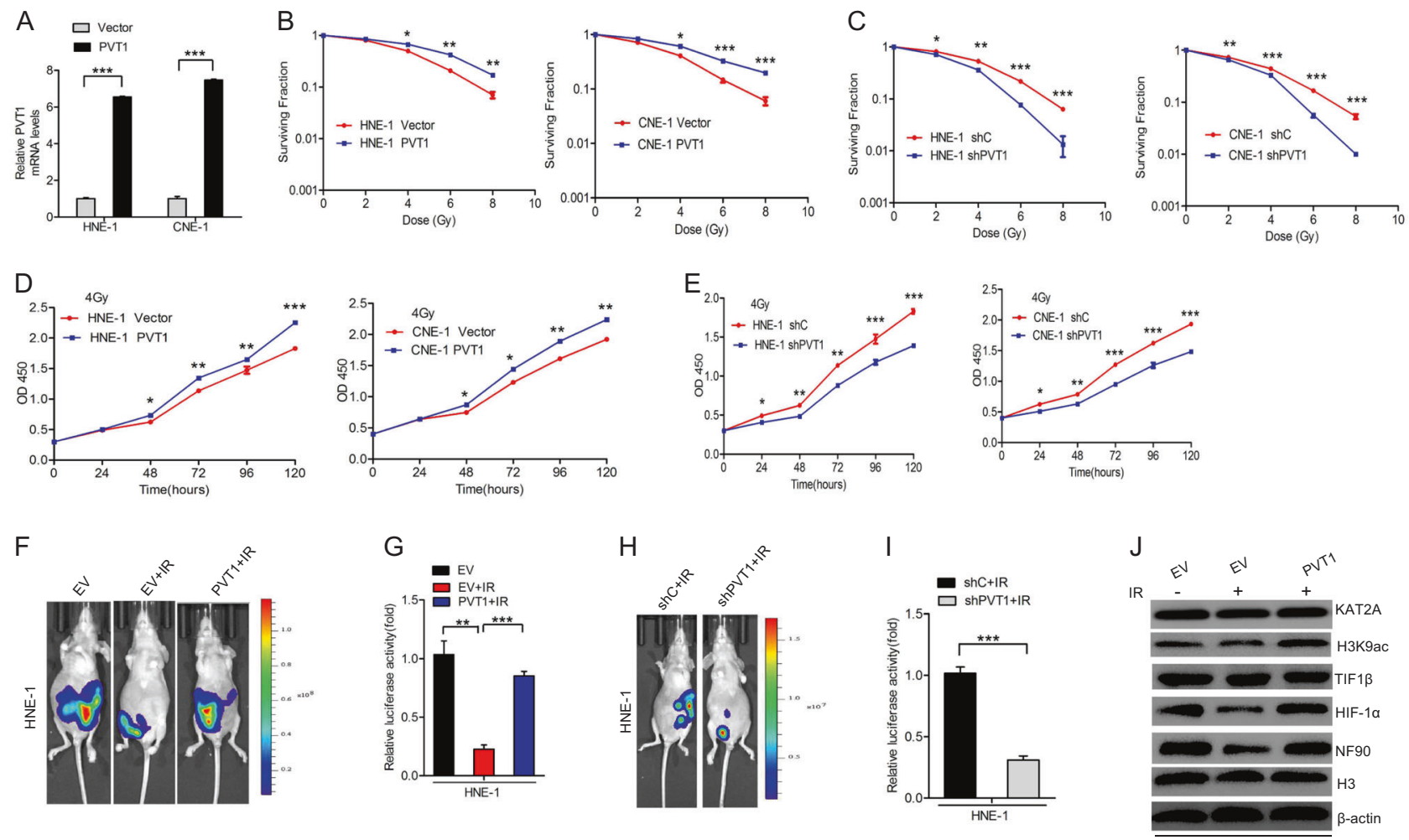

K HNE-1 RIP-qPCR, PVT1

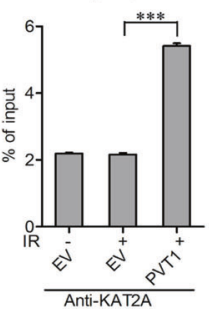

HNE-1

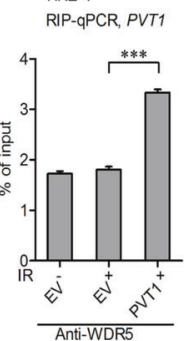

$\mathrm{L}$

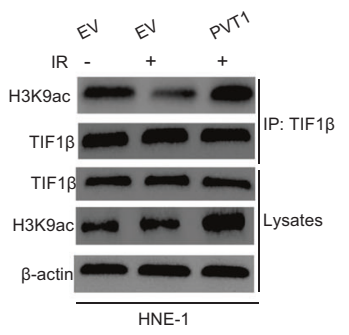

Fig. 9 PVT1 suppresses the radiosensitivity of NPC cell lines by increasing the interaction between TIF1 $\beta$ and H3K9ac. a Effects of PVT1 overexpression in HNE-1 and CNE-1 NPC cells. Colony formation assays of PVT1 knockdown-(b) and overexpression-(c) mediated radiosensitivity in HNE-1 and CNE-1 NPC cells. Cell proliferation assays of PVT1 knockdown-(d) and overexpression-(e) mediated radiosensitivity in HNE-1 and CNE-1 NPC cells. f Representative bioluminescence images of PVT1 overexpression-restored radiation-inhibited HNE-1 subcutaneous tumor generation. Mice were imaged at 3-4 weeks after implantation. Data were from two independent experiments with five mice per group with similar results. $g$ Quantification of

transcribed from the interleukin enhancer binding factor 3 gene. A growing body of evidence show that NF90 regulates mRNA stability and plays a vital role in RNA metabolism $[39,40]$. Thus, we hypothesized that PVT1 might increase HIF-1 $\alpha$ mRNA stability via activating NF90 transcription. Indeed, the ChIP assay confirmed that PVT1 knockdown suppressed NF90 transcription and NF90 overexpression rescued PVT1 depletion-inhibited HIF-1 $\alpha$ mRNA stability. Taken together, our data show that PVT1 regulates NPC cell proliferation through HIF-1 $\alpha$ stabilization.
M

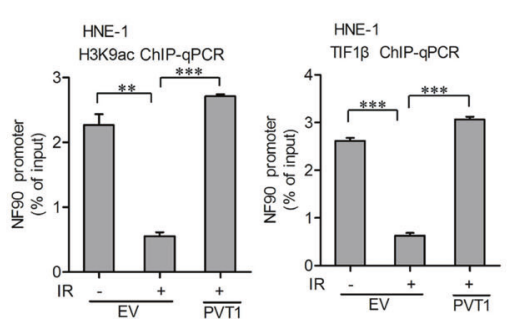

the bioluminescence activity in $\mathbf{f}$. $\mathbf{h}$ Representative bioluminescence images of PVT1 knockdown-induced radiation-inhibited HNE-1 subcutaneous tumor generation. i Quantification of the bioluminescence activity in $\mathbf{h}$. $\mathbf{j}$ Overexpression of PVT1 rescues radiation-inhibited HIF$1 \alpha$ and NF90 expression. $\mathbf{k}$ The RIP assay of radiation has no effect on the association of KAT2A or WDR5, and PVT1. I Overexpression of PVT1 rescues radiation-inhibited the interaction between TIF1 $\beta$ and H3K9ac. m Effects of PVT1 overexpression on radiation-inhibited the association of KAT2A or WDR5 with the NF90 promoter. Error bars \pm SD. $* P<0.05$. $* * P<0.01$. $* * * P<0.001$. Data are representative from three independent experiments

Our results further revealed that PVT1 serves as a scaffold for the chromatin modification factor KAT2A, which stabilizes HIF-1 $\alpha$ through H3K9ac/TIF1 $\beta$ complex-mediated NF90 transcriptional activation. Previous work has shown that the lncRNAs GClnc1 and GCAWKR can serve as scaffolds for KAT2A and WDR5 in gastric cancer, and can thereby serve as positive regulators of this enzyme [5, 41]. The IncRNA NEAT1 also interacts with KAT2A to promote deterioration of hepatocellular carcinoma [6]. However, the relationship of PVT1 and KAT2A has not previously been 


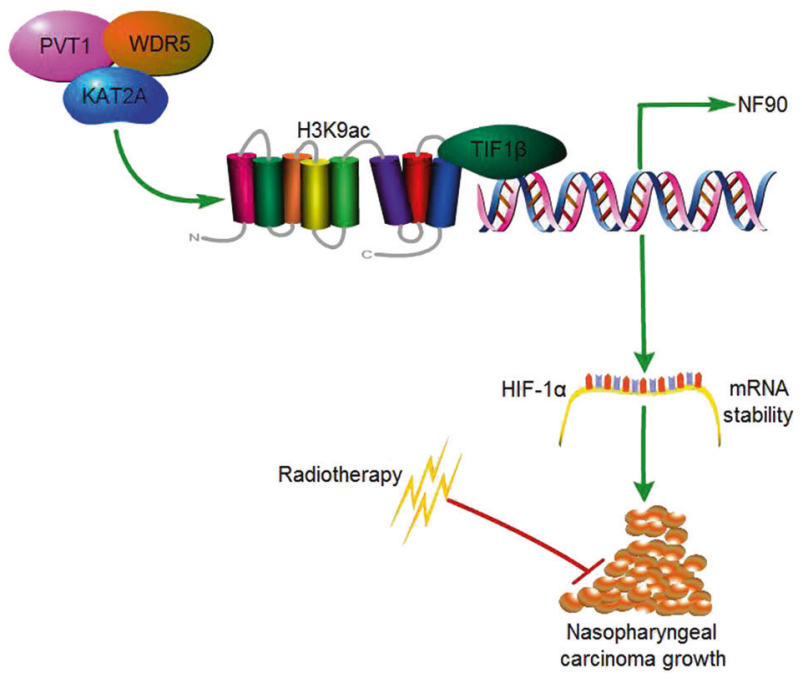

Fig. 10 A working model for PVT1-regulated NPC tumorigenesis. PVT1 acts as a scaffold of KAT2A and WDR5, and KAT2A promotes H3K9 acetylation and association with TIF1 $\beta$, leading to increased NF90 transcription and HIF-1 $\alpha$ stabilization, thus resulting in enhanced NPC tumorigenesis

reported. Here, we found that PVT1 can interact with KAT2A, and thereby promotes enhanced $\mathrm{H} 3 \mathrm{~K} 9$ acetylation in NPC cells. KAT2A-mediated H3K9 acetylation in turn induces the binding of TIF1 $\beta$ to chromatin. Consistent with previous studies of HIF-1 $\alpha$ mRNA stability [30], our data demonstrate that overexpression of KAT2A or TIF1 $\beta$ upregulate PVT1-mediated NF90 transcriptional activation in NPC cells in order to increase HIF-1 $\alpha$ mRNA stability, while overexpression of KAT2A acetyltransferase activity-deficient mutants or TIF1 $\beta$ mutants lacking H3K9ac binding sites does not. Our results thus demonstrate that PVT1 serves as a scaffold for KAT2A-mediated regulation of HIF- $1 \alpha$ mRNA stability in a manner dependent on its acetyltransferase activity and on H3K9ac/TIF1 $\beta$ complex-mediated NF90 transcriptional activation.

Radiation is a primary treatment for NPC [20]. PVT1mediated suppression of tumor radiosensitivity has been reported in non-small cell lung cancer [42], and NPC [20], respectively. However, the specific underlying signaling pathways were not well characterized. Our data suggest that radiation inhibits NPC cell proliferation via abrogating the binding between $\mathrm{H} 3 \mathrm{~K} 9 \mathrm{ac}$ and TIF $1 \beta$, and that this binding is restored by overexpression of PVT1, whereas the binding between PVT1 and KAT2A is unaffected.

In summary, our data identify PVT1 as a potential treatment target in individuals with NPC. Our results further demonstrate a mechanism whereby PVT1 promotes NPC cell proliferation via a KAT2A/H3K9ac/TIF1//NF90/HIF$1 \alpha$ signaling pathway. Utilization of these results clinically may allow for better personalized radiation dosing based on PVT1 expression in NPC patients.

\section{Materials and methods}

\section{Clinical specimens}

Ten freshly frozen normal nasopharyngeal samples and ten NPC biopsy samples were collected from Zhongshan City People's Hospital. In addition, 100 formalin-fixed paraffinembedded NPC tissues were also obtained from Zhongshan City People's Hospital. between January 2010 and December 2015. All patients had not received radiotherapy and chemotherapy before biopsy. Written patient consent and approval from the Institutional Ethical Review Boards of the Meizhou People's Hospital were obtained. The usage of specimens were carried out according to the approved guidelines of the Zhongshan City People's Hospital.

\section{Cell culture}

Human NPC cell lines (SUNE-1, C666-1,CNE-2, CNE-1, and HNE-1) were obtained from Cell Bank of the Chinese Scientific Academy (Shanghai, China), and were cultured in Dulbecco's modified Eagle's medium (Invitrogen, Carlsbad, CA) supplemented with $10 \%$ fetal bovine serum. NP69 was also purchased from Cell Bank of the Chinese Academy of Sciences (Shanghai, China), and was cultured in DMEM/F12 (Invitrogen, Carlsbad, CA) supplemented with $5 \%$ horse serum, $20 \mathrm{ng} / \mathrm{ml}$ epidermal growth factor, $0.5 \mu \mathrm{g} / \mathrm{ml}$ hydrocortisone, $100 \mathrm{ng} / \mathrm{ml}$ cholera toxin, $10 \mu \mathrm{g} / \mathrm{ml}$ insulin, and $100 \mu \mathrm{g} / \mathrm{ml}$ penicillin-streptomycin. Cells were cultured in a humidified incubator in a $5 \% \mathrm{CO}_{2}$ atmosphere at $37^{\circ} \mathrm{C}$. Hypoxic conditions were achieved with a hypoxia chamber (Billups-Rothenberg) flushed with a gas mixture of $1 \% \mathrm{O}_{2}, 5 \% \mathrm{CO}_{2}$ and $94 \% \mathrm{~N}_{2}$.

\section{RNA extraction, reverse transcription, and quantitative PCR}

TRIzol reagent (Invitrogen) was utilized to isolate total RNA from NCP tissues and cells. The RNeasy FFPE kit (QIAGEN GmbH) was utilized to extract total RNA from FFPE NPC tissues. The RNA quality and amount were evaluated by a NanoDrop 3300 spectrophotometer (Thermo Scientific). Reverse transcriptase (Promega) was utilized to perform reverse transcription. SYBR Green qPCR SuperMix-UDG (Thermo Fisher) was utilized to conduct Quantitative real-time PCR. $\beta$-actin was used as the normalization control. Specific primers are shown in Supplenentary Table S1.

\section{Western blotting}

Western blot was conducted as we previously described [43]. The antibodies for western blotting are listed as 
follows: $\beta$-actin (\#4970, 1:1000, Cell Signaling Technology), KAT2A (\#3305S, 1:1000, Cell Signaling Technology), HIF-1 $\alpha$ (\#36169, 1:1000, Cell Signaling Technology), H3K9ac (\#9649, 1:1000, Cell Signaling Technology), Histone H3 (\#4499, 1:1000, Cell Signaling Technology), TIF1 $\gamma$ (\#13387, 1:1000, Cell Signaling Technology), TIF1 $\beta$ (\#4124, 1:1000, Cell Signaling Technology), TIF1 $\alpha$ (ab38264, 1:1000, Abcam), NCOA1 (\#20301, 1:1000, Cell Signaling Technology), NCOA2 (\#96687, 1:1000, Cell Signaling Technology), NCOA3 (\#2126, 1:1000, Cell Signaling Technology), NF90 (sc-377406, 1:500, Santa Cruz Biotechnology), WDR5 (\#13105,1:1000, Cell Signaling Technology), KAT2B (\#3378, 1:1000, Cell Signaling Technology), KAT5 (\#12058,1:1000, Cell Signaling Technology), KAT8 (\#46862, 1:1000, Cell Signaling Technology), and KAT6A (ABP59003, 1:1000, Abbkine).

\section{ChIP-qPCR}

The chromatin immunoprecipitation kit (Millipore-17-408) was utilized to perform the ChIP assay according to the manufacturer's instruction. The immunoprecipitated DNAs were quantified through quantitative real-time PCR. Specific primers are shown in Supplenentary Table S1.

\section{In situ hybridization}

The in situ detection of PVT1 was performed on paraffinembedded sections using the RNAscope 2.5 HD Detection Reagent-BROWN kit (ACDBio). The specific PVT1 probes were made by ACDBio.

\section{Construction of vectors}

The cDNA encoding PVT1, KAT2A, TIF1 $\beta$, and NF90 were amplified from NP69 cells and sequenced, and then subcloned into the pcDNA3 vector (Invitrogen), subsequently named pCDNA3-PVT1, pCDNA3-KAT2A, pCDNA3-TIF1 $\beta$, and pCDNA3-NF90. pLVX-PVT1, pLVX-KAT2A, pLVX-TIF1 $\beta$, and pLVX-NF90 was generated from pCDNA3-PVT1, pCDNA3-KAT2A, pCDNA3TIF1 $\beta$, and pCDNA3-NF90, respectively. NF90 promoter was PCR-amplified from NP69 cells and sequenced, and then subcloned into pGL3 vector (Promega). A Quik Change Site-Directed Mutagenesis Kit (Stratagene) was used for point mutations. shRNAs were designed as follows: PVT1 (shPVT1-1 target sequence: 5'-GCCATCATG ATGGTACTTTAA-3'; shPVT1-2 target sequence 5'-GC CA GGACACTG AGATTTGGA-3'); KAT2A (shKAT2 A-1 target sequence: 5'-CGTGCTGTCACCTCGAATG A-3'; shKAT2A-2 target sequence 5'-TCATGTCTGT TCACAAGGAA-3'); TIF1 $\beta$ (shTIF1 $\beta$ target sequence: $5^{\prime}$-T CTGTTCTCTGTCCTCTCGAGAGGA
CAGAGAACAGAGCCAGG-3'); NF90 (shNF90-1 target sequence: 5'-GTGCTGGTTCCAACAAAA-3'; shNF90-2 target sequence $5^{\prime}$-AGTCGT GGAAAGCCTAAGA-3'). shRNAs was cloned into lentiviral expression vector pLL3.7.

\section{Colony formation and CCK8 assays}

For the colony formation assay, 600 cells in $2 \mathrm{ml}$ medium were seeded into six-well plates and cultured for 6 or 11 days. Cell colonies were then successively fixed, stained, and counted. For the CCK8 assay, $2 \times 10^{3}$ cells in $100 \mu \mathrm{l}$ medium solution were cultured in 96-well plates. Then, a $10 \mu \mathrm{l} \mathrm{CCK8}$ reagent (DOJINDO) was added to each well. After the 96well plates were incubated for $2 \mathrm{~h}$ at $37{ }^{\circ} \mathrm{C}$, we then measure the absorbance at $450 \mathrm{~nm}$ for each sample well.

\section{RNA immunoprecipitation (RIP) and RNA pull-down assays}

RIP and RNA pull-down assays were performed as we previously described [44]. Antibodies used for RIP are displayed as follows: WDR5 (\#13105,1:50, Cell Signaling Technology), KAT2B (\#3378, 1:50, Cell Signaling Technology), KAT5 (\#12058,1:50, Cell Signaling Technology), KAT8 (\#46862, 1:50, Cell Signaling Technology), KAT6A (ABP59003, 1:100, Abbkine), and KAT2A (\#3305S, 1:50, Cell Signaling Technology).

\section{RNA sequencing}

Total RNA was extracted from HNE-1 transfected with shPVT1 or shC control and three free-frozen NPC tissues and corresponding normal nasopharyngeal specimens. The RNA quality was measured through Agilent Bioanalyzer 2100 (Agilent technologies). Qualified RNA was then purified by RNA Clean XP Kit (A63987, Beckman) and Qiagen RNase-Free DNase Set (79254, QIAGEN).

Next, we constructed Illumina sequencing libraries according to the instruction. Briefly, we extracted noncoding and coding RNA from $5 \mu \mathrm{g}$ of purified total RNA using a Ribo-Zero rRNA Removal Kit. After isolation, we then eluted and fragmented the RNA samples at $94{ }^{\circ} \mathrm{C}$ for 8 min with Elute-Prime-Fragment mix. Subsequently, we synthesized the first-strand cDNA using Invitrogen SuperScript II. Then, Second-Strand Marking Master Mix mixed with the product was incubated at $16^{\circ} \mathrm{C}$ for $1 \mathrm{~h}$ to synthesize the second-strand cDNA. After $3^{\prime}$ ends adenylation, we ligated the DNA fragments with TruSeq adapters and the product was amplified using TruSeq PCR primers. Then, the libraries were loaded on cBot (Illumina) to generate a cluster and subsequently sequenced on the HiSeq $\mathrm{X}$ ten (Illumina) platform at the Ling En biotechnological Corporation (Shang hai). 
RNA-seq reads were mapped to the human reference genome sequence (hg38) assembly by Hisat2 (Version 2.1.0). Stringtie (version 1.3.3) was used to count the number of fragments of each known gene downloaded from Ensembl (Homo sapiens GRCh38). After mapping, we removed genes with fewer than ten fragments in all of the samples and identified differentially expressed genes using $\mathrm{R} /$ Bioconductor package edge $\mathrm{R}$ (version 3.0.8). Thresholds of $P<0.05$ and fold change $\geq 1.5$ were used to identify differentially expressed genes.

The differentially expressed genes were used to perform KEGG (Kyoto Encyclopedia of Genes and Genomes) pathway analysis and GO analysis using DAVID software (https://david.ncifcrf.gov/). Two-sided or Bonferronicorrected Fisher's exact tests were used to calculate the $P$-values of KEGG pathways or GO terms, respectively. A threshold of $P<0.05$ and $\mathrm{FDR} \leq 0.25$ was used to select significant items. RNA data are available at the NCBI BioProject database (http://www.ncbi.nlm.nih.gov/ bioproject) under Bioproject ID: PRJNA548225.

\section{Tumorigenesis studies}

Four- to five-week-old female BALB/c nude mice (SLAC) were randomly divided into per group, and then HNE-1 cells $\left(2 \times 10^{6}\right)$ were implanted subcutaneously into each mice. All animal experiments procedures were performed according to guidelines approved by Zhejiang Provincial People's Hospital the Guidance of Institutional Animal Care and Use Committee. As previously described [17], HNE-1 cells were transduced with a luciferase reporter plasmids. Tumor volumes were measured using the largest tumor cross section for each samples in vivo using luciferase activity after injection of D-luciferin. The IVIS Lumina imaging station (Caliper Life Sciences) was used for bioluminescence imaging. Different investigators independently performed mice allocation, surgery and the outcome assessing.

\section{Immunohisochemical staining of human NPC specimens}

Human NPC specimens including paraffin-embeddededidentified NPC tissues and corresponding normal nasopharyngeal specimens were obtained from Zhongshan City People's Hospital. The specimens were stained with antibodies against KAT2A (1:50) and WDR5 (1:50). Immunohisochemical staining was scored as previously described [40].

\section{Statistical analysis}

The data were conducted using the GraphPad Prism version 5.0. The cumulative survival rate was evaluated using the Kaplan-Meier method. The statistical analyses of data from experimental groups was determined by multiple comparison tests. $* P<0.05$ was considered statistically significant.

Funding This study was supported in part by grants from National Natural Science Foundation of China (Grant number: 81672430 to XM); Zhejiang Provincial Nature Science Foundation of China (Grant number: LY18H160035 to XL); Zhejiang Provincial People's Hospital Scientific Research Returned Foundation for The Excellent Youth (Grant number: ZRY2018B002 to JT); Zhejiang Provincial Nature Science Foundation of China (Grant number: LY19H160032 to SZ); National Natural Science Foundation of China (Grant number: 81602671 to SZ)

\section{Compliance with ethical standards}

Conflict of interest The authors declare that they have no conflict of interest.

Publisher's note: Springer Nature remains neutral with regard to jurisdictional claims in published maps and institutional affiliations.

Open Access This article is licensed under a Creative Commons Attribution 4.0 International License, which permits use, sharing, adaptation, distribution and reproduction in any medium or format, as long as you give appropriate credit to the original author(s) and the source, provide a link to the Creative Commons license, and indicate if changes were made. The images or other third party material in this article are included in the article's Creative Commons license, unless indicated otherwise in a credit line to the material. If material is not included in the article's Creative Commons license and your intended use is not permitted by statutory regulation or exceeds the permitted use, you will need to obtain permission directly from the copyright holder. To view a copy of this license, visit http://creativecommons. org/licenses/by/4.0/.

\section{References}

1. Torre LA, Bray F, Siegel RL, Ferlay J, Lortet-Tieulent J, Jemal A. Global cancer statistics, 2012. CA: Cancer J Clin. 2015;65:87-108.

2. Wei KR, Zheng RS, Zhang SW, Liang ZH, Li ZM, Chen WQ. Nasopharyngeal carcinoma incidence and mortality in China, 2013. Chin J Cancer. 2017;36:90.

3. Feng XP, Yi H, Li MY, Li XH, Yi B, Zhang PF, et al. Identification of biomarkers for predicting nasopharyngeal carcinoma response to radiotherapy by proteomics. Cancer Res. 2010;70:3450-62.

4. Zeng Z, Fan S, Zhang X, Li S, Zhou M, Xiong W, et al. EpsteinBarr virus-encoded small RNA 1 (EBER-1) could predict good prognosis in nasopharyngeal carcinoma. Clin Transl Oncol. 2016;18:206-11.

5. Sun TT, He J, Liang Q, Ren LL, Yan TT, Yu TC, et al. LncRNA GClnc1 promotes gastric carcinogenesis and may act as a modular scaffold of WDR5 and KAT2A complexes to specify the histone modification pattern. Cancer Discov. 2016;6:784-801.

6. Ling ZA, Xiong DD, Meng RM, Cen JM, Zhao N, Chen G, et al. LncRNA NEAT1 promotes deterioration of hepatocellular carcinoma based on in vitro experiments, data mining, and RT-qPCR analysis. Cell Physiol Biochem. 2018;48:540-55.

7. Cory S, Graham M, Webb E, Corcoran L, Adams JM. Variant $(6 ; 15)$ translocations in murine plasmacytomas involve a chromosome 15 locus at least $72 \mathrm{~kb}$ from the c-myc oncogene. EMBO J. 1985;4:675-81. 
8. Webb E, Adams JM, Cory S. Variant $(6 ; 15)$ translocation in a murine plasmacytoma occurs near an immunoglobulin kappa gene but far from the myc oncogene. Nature. 1984;312:777-9.

9. Cui D, Yu CH, Liu M, Xia QQ, Zhang YF, Jiang WL. Long noncoding RNA PVT1 as a novel biomarker for diagnosis and prognosis of non-small cell lung cancer. Tumour Biol. 2016;37:4127-34.

10. Wan L, Sun M, Liu GJ, Wei CC, Zhang EB, Kong R, et al. Long noncoding RNA PVT1 promotes non-small cell lung cancer cell proliferation through epigenetically regulating LATS2 expression. Mol Cancer Ther. 2016;15:1082-94.

11. Yang YR, Zang SZ, Zhong CL, Li YX, Zhao SS, Feng XJ. Increased expression of the IncRNA PVT1 promotes tumorigenesis in non-small cell lung cancer. Int $\mathbf{J}$ Clin Exp Pathol. 2014;7:6929-35.

12. Takahashi Y, Sawada G, Kurashige J, Uchi R, Matsumura T, Ueo $\mathrm{H}$, et al. Amplification of PVT-1 is involved in poor prognosis via apoptosis inhibition in colorectal cancers. Br J cancer. 2014; 110:164-71.

13. Yuan CL, Li H, Zhu L, Liu Z, Zhou J, Shu Y. Aberrant expression of long noncoding RNA PVT1 and its diagnostic and prognostic significance in patients with gastric cancer. Neoplasma. 2016;63:442-9.

14. Zhao J, Du P, Cui P, Qin Y, Hu C, Wu J, et al. LncRNA PVT1 promotes angiogenesis via activating the STAT3/VEGFA axis in gastric cancer. Oncogene. 2018;37:4094-109.

15. Fan H, Zhu JH, Yao XQ. Long non-coding RNA PVT1 as a novel potential biomarker for predicting the prognosis of colorectal cancer. Int J Biol Markers. 2018. https://doi.org/10.1177/ 1724600818777242.

16. Huang C, Liu S, Wang H, Zhang Z, Yang Q, Gao F. LncRNA PVT1 overexpression is a poor prognostic biomarker and regulates migration and invasion in small cell lung cancer. Am J Transl Res. 2016;8:5025-34.

17. Tang J, Li Y, Sang Y, Yu B, Lv D, Zhang W, et al. LncRNA PVT1 regulates triple-negative breast cancer through KLF5/betacatenin signaling. Oncogene. 2018;37:4723-34.

18. Kong R, Zhang EB, Yin DD, You LH, Xu TP, Chen WM, et al. Long noncoding RNA PVT1 indicates a poor prognosis of gastric cancer and promotes cell proliferation through epigenetically regulating p15 and p16. Mol Cancer. 2015;14:82.

19. Xu MD, Wang Y, Weng W, Wei P, Qi P, Zhang Q, et al. A positive feedback loop of lncRNA-PVT1 and FOXM1 facilitates gastric cancer growth and invasion. Clin Cancer Res. 2017;23:2071-80.

20. He Y, Jing Y, Wei F, Tang Y, Yang L, Luo J, et al. Long noncoding RNA PVT1 predicts poor prognosis and induces radioresistance by regulating DNA repair and cell apoptosis in nasopharyngeal carcinoma. Cell Death Dis. 2018;9:235.

21. Sengupta S, den Boon JA, Chen IH, Newton MA, Dahl DB, Chen $\mathrm{M}$, et al. Genome-wide expression profiling reveals EBVassociated inhibition of MHC class I expression in nasopharyngeal carcinoma. Cancer Res. 2006;66:7999-8006.

22. Bo H, Gong Z, Zhang W, Li X, Zeng Y, Liao Q, et al. Upregulated long non-coding RNA AFAP1-AS1 expression is associated with progression and poor prognosis of nasopharyngeal carcinoma. Oncotarget. 2015;6:20404-18.

23. Kurokawa R. Promoter-associated long noncoding RNAs repress transcription through a RNA binding protein TLS. Adv Exp Med Biol. 2011;722:196-208.

24. Fournier M, Orpinell M, Grauffel C, Scheer E, Garnier JM, Ye T, et al. KAT2A/KAT2B-targeted acetylome reveals a role for PLK4 acetylation in preventing centrosome amplification. Nat Commun. 2016;7:13227.

25. Ellinghaus P, Heisler I, Unterschemmann K, Haerter M, Beck H, Greschat S, et al. BAY 87-2243, a highly potent and selective inhibitor of hypoxia-induced gene activation has antitumor activities by inhibition of mitochondrial complex I. Cancer Med. 2013;2:611-24.
26. Helbig L, Koi L, Brüchner K, Gurtner K, Hess-Stumpp H, Unterschemmann K, et al. BAY 87-2243, a novel inhibitor of hypoxia-induced gene activation, improves local tumor control after fractionated irradiation in a schedule-dependent manner in head and neck human xenografts. Radiat Oncol. 2014;9:207.

27. Tsai WW, Wang Z, Yiu TT, Akdemir KC, Xia W, Winter S, et al. TRIM24 links a non-canonical histone signature to breast cancer. Nature. 2010;468:927-32.

28. Groner AC, Cato L, de Tribolet-Hardy J, Bernasocchi T, Janouskova H, Melchers D, et al. TRIM24 Is an oncogenic transcriptional activator in prostate cancer. Cancer Cell. 2016;29:846-58.

29. Lv D, Jia F, Hou Y, Sang Y, Alvarez AA, Zhang W, et al. Histone acetyltransferase KAT6A upregulates PI3K/AKT signaling through TRIM24 binding. Cancer Res. 2017;77:6190-201.

30. Wen X, Liu X, Mao YP, Yang XJ, Wang YQ, Zhang PP, et al. Long non-coding RNA DANCR stabilizes HIF-1alpha and promotes metastasis by interacting with NF90/NF45 complex in nasopharyngeal carcinoma. Theranostics. 2018;8:5676-89.

31. Wu X, Ruan L, Yang Y, Mei Q. Identification of crucial regulatory relationships between long non-coding RNAs and proteincoding genes in lung squamous cell carcinoma. Mol Cell probes. 2016;30:146-52.

32. Gao YL, Zhao ZS, Zhang MY, Han LJ, Dong YJ, Xu B. Long noncoding RNA PVT1 facilitates cervical cancer progression via negative regulating of miR-424. Oncol Res. 2017;25:1391-8.

33. Cui M, Chang Y, Fang QG, Du W, Wu JF, Wang JH, et al. Noncoding RNA Pvt1 promotes cancer stem cell-like traits in nasopharyngeal cancer via inhibiting miR-1207. Pathology Oncol Res. 2018. https://doi.org/10.1007/s12253-018-0453-1.

34. Yeh SH, Liu RS, Wu LC, Yang DJ, Yen SH, Chang CW, et al. Fluorine-18 fluoromisonidazole tumour to muscle retention ratio for the detection of hypoxia in nasopharyngeal carcinoma. Eur $\mathbf{J}$ Nucl Med. 1996;23:1378-83.

35. Hong B, Lui VW, Hashiguchi M, Hui EP, Chan AT. Targeting tumor hypoxia in nasopharyngeal carcinoma. Head Neck. 2013;35:133-45.

36. Rankin EB, Giaccia AJ. Hypoxic control of metastasis. Science. 2016;352:175-80.

37. Higgins DF, Kimura K, Bernhardt WM, Shrimanker N, Akai Y, Hohenstein B, et al. Hypoxia promotes fibrogenesis in vivo via HIF-1 stimulation of epithelial-to-mesenchymal transition. J Clin Investig. 2007;117:3810-20.

38. Su X, Li G, Liu W. The long noncoding RNA cancer susceptibility candidate 9 promotes nasopharyngeal carcinogenesis via stabilizing HIF1alpha. DNA Cell Biol. 2017;36:394-400.

39. Schmidt T, Friedrich S, Golbik RP, Behrens SE. NF90-NF45 is a selective RNA chaperone that rearranges viral and cellular riboswitches: biochemical analysis of a virus host factor activity. Nucleic Acids Res. 2017;45:12441-54.

40. Castella S, Bernard R, Corno M, Fradin A, Larcher JC. Ilf3 and NF90 functions in RNA biology. Wiley Interdiscip Rev RNA. 2015;6:243-56.

41. Ma M, Zhang Y, Weng M, Hu Y, Xuan Y, Hu Y, et al. lncRNA GCAWKR promotes gastric cancer development by scaffolding the chromatin modification factors WDR5 and KAT2A. Mol Ther. 2018;26:2658-68.

42. Wu D, Li Y, Zhang H, Hu X. Knockdown of Lncrna PVT1 enhances radiosensitivity in non-small cell lung cancer by sponging mir-195. Cell Physiol Biochem. 2017;42:2453-66.

43. Tang J, Zhong G, Wu J, Chen H, Jia Y. SOX2 recruits KLF4 to regulate nasopharyngeal carcinoma proliferation via PI3K/AKT signaling. Oncogenesis. 2018;7:61.

44. Tang J, Zhong G, Zhang H, Yu B, Wei F, Luo L, et al. LncRNA DANCR upregulates PI3K/AKT signaling through activating serine phosphorylation of RXRA. Cell Death Dis. 2018;9:1167. 


\section{Affiliations}

\section{Ying Wang ${ }^{1} \cdot$ Wanyuan Chen $^{2} \cdot{\text { Jiayan } \operatorname{Lian}^{3} \cdot \text { Haibo Zhang }}^{1} \cdot$ Bo Yu $^{4} \cdot$ Minjun Zhang $^{5} \cdot$ Fangqiang Wei $^{6}$.

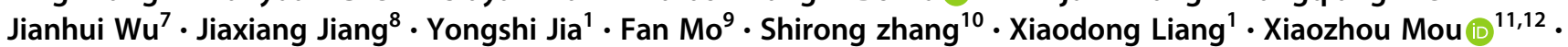 Jianming Tang ${ }^{1}$}

1 Department of Radiation Oncology, Zhejiang Provincial People's Hospital, People' s Hospital of Hangzhou Medical College, Hangzhou 310014 Zhejiang, PR China

2 Department of Pathology, Zhejiang Provincial People's Hospital, People's Hospital of Hangzhou Medical College, Hangzhou 310014 Zhejiang, PR China

3 Department of Pathology, the 7th affiliated hospital of Sun Yat-Sen University, Shenzhen 510275 Guandong, PR China

4 State Key Laboratory of Oncogenes and Related Genes, Renji-Med X Clinical Stem Cell Research Center, Ren Ji Hospital, School of Medicine, Shanghai Jiao Tong University, 200127 Shanghai, PR China

5 Graduate Department, Bengbu Medical College, Bengbu 233000 Anhui, PR China

6 Department of Hepatobiliary and Pancreatic Surgery, Zhejiang Provincial People's Hospital, People's Hospital of Hangzhou Medical College, Hangzhou 310014 Zhejiang, PR China

7 Department of The Otolaryngology, Zhongshan City People's
Hospital, Zhongshan Affiliated Hospital of Sun Yan-sen University, Zhongshan 528403 Guangdong, PR China

8 Department of Cardiology, Zhejiang Provincial People's Hospital, People's Hospital of Hangzhou Medical College, Hangzhou 310014 Zhejiang, PR China

9 Vancouver Prostate Centre, University of British Columbia, 2660 Oak St., Vancouver, BC V6H 3Z6, Canada

10 Translational medicine centre, Affiliated Hangzhou First People's Hospital, Zhejiang University School of Medicine, Hangzhou 310006 Zhejiang, PR China

11 Key Laboratory of Tumor Molecular Diagnosis and Individualized Medicine of Zhejiang Province, Zhejiang Provincial People's Hospital, People's Hospital of Hangzhou Medical College, Hangzhou 310014 Zhejiang, PR China

12 Clinical Research Institute, Zhejiang Provincial People's Hospital, People's Hospital of Hangzhou Medical College, Hangzhou 310014 Zhejiang, PR China 